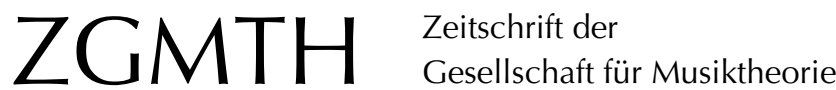

Noll, Thomas / Nestke, Andreas (2003/05): Die Apperzeption von Tönen. ZGMTH $1-$ 2/1/1, 107-135. https://doi.org/10.31751/486

(c) 2003/05 Thomas Noll, Andreas Nestke

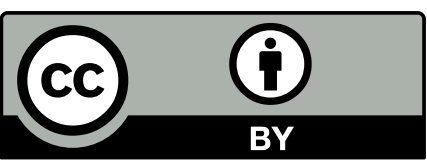

Dieser Text erscheint im Open Access und ist lizenziert unter einer Creative Commons Namensnennung 4.0 International Lizenz.

This is an open access article licensed under a

Creative Commons Attribution 4.0 International License.

veröffentlicht / first published: 01/01/2003

zuletzt geändert / last updated: 15/09/2009 


\title{
Die Apperzeption von Tönen
}

\author{
Thomas Noll und Andreas Nestke, \\ Institut für Informatik, Technische Universität Berlin
}

Die vorliegende Arbeit ist der Modellierung von Tönen und Tonverwandtschaften gewidmet. Dabei bezeichnet der Begriff der >Apperzeption den Akt des Erreichens eines Zieltonorts von einem Ausgangstonort aus. Anlaß solcher Apperzeptionen sind jeweils Tonintervalle, die wir Tonvektoren nennen, um ihren Impulscharakter zu betonen. Die Wahl des philosophisch beladenen Begriffes ${ }^{1}$ mag nicht unproblematisch sein, zumal von uns nicht der Anspruch erhoben wird, das konkrete subjektive Erleben - sei es beim aktiven musikalischen Hören oder beim inneren Vorstellen ${ }^{2}$ etwa von Tonfortschreitungen - zu erfassen. Dennoch halten wir es für möglich, daß sich das gefundene Modell als Schlüssel für ein tieferes Verständnis musikalischen Denkens erweisen könnte. Zugunsten dieser These läßt sich ein musiktheoretisches Argument vorbringen: Das Modell liefert eine konsistente, in sich geschlossene Antwort auf eine Reihe offener Fragen zum Tonsystem. Unser Versuch, ein mathematisches Modell der Apperzeption zu finden, führte uns zu einer neuen Sicht auf das sEulersche Tonnetzı, wie man es in vielen Lehrbüchern der Harmonielehre findet. Der Tonraum unseres Modells - auch bekannt als Antide-Sitter-Raum $\mathrm{adS}_{3}$ - besitzt eine natürliche Metrik (im Sinne der pseudo-riemannschen Geometrie), die ähnlich der Lorentz-Metrik in der allgemeinen Relativitätstheorie entartet und die konstante Krümmung -1 hat.

\section{Offene Fragen zum Tonsystem}

Dreh- und Angelpunkt unseres Theorieentwurfs ist die Auflösung der verbreiteten und irreführenden Identifikation von Intervallen und Tonpaaren. Den großen Nutzen, den eine Sensibilisierung der Theorie gegenüber diesem Punkt bringen kann, zeigt Guerino Mazzolas Modellierung des zweistimmigen Kontrapunkts im Rahmen seiner »Geometrie der Töne« (Mazzola 1990).

Der vorliegende Theorieansatz übernimmt Mazzolas geometrische Auffassung von Intervallen im Sinne von sTonvektoren

1 Nach unserem Verständnis handelt es sich bei dem vorgelegten Theorieentwurf um eine rein musiktheoretische Arbeit. Den offenbar gegenbenen Bezug zu Leibniz' `Monadologie` herauszuarbeiten, würde ein eigenes Forschungsprojekt bedeuten. Tonapperzeption bezeichnet daher im Rahmen dieses Beitrages einen autonomen musiktheoretischen Forschungsbereich jenseits der Psychoakustik.

2 Hugo Riemanns »Ideen zu einer Lehre von den Tonvorstellungen« sind eine wichtige Quelle für den vorgelegten Theorieansatz. Dennoch erlaubt uns der gegenwärtige Entwicklungsstand der Theorie nicht, die modellhaften Apperzeptionsakte bereits zur Beschreibung individueller Denkvorgänge heranzuziehen. 
im Sinne geordneter Tonpaare um den zentralen Begriff der >Tonapperzeption «. ${ }^{3}$ Indem wir Apperzeptionen als Akte des Erreichens von Zieltönen von Ausgangstönen aus zu den zentralen Gegenständen der Theorie machen, wollen wir zugleich suggerieren, diese Akte letztlich auch als die selementaren`Gegenstände der Musiktheorie anzusehen. Dabei ist es nicht erforderlich, eine psychologische Realitätsebene zu betrachten. Vielmehr gleicht unsere Schwerpunktverlagerung dem Vorgehen eines Linguisten, der Verben als elementarer erachtet als Substantive. Tonverwandtschaft betrifft aus Sicht der strukturalistischen Semiotik die Paradigmatik von Tonbeziehungen. Während die mengentheoretisch orientierte Semiotik der Paradigmatik eine passive Rolle zuweist, konstatieren wir eine aktive Paradigmatik, deren Grundelemente (paradigmatische) Apperzeptionen sind.

\subsection{Intension und Extension von Tonverwandtschaften}

In der Behandlung von Tonverwandtschaften begegnen uns in der Musiktheorie in verschiedenerlei Ausprägungen zwei Grundmodelle, deren Zusammenhang wir diskutieren wollen: `Gitter und `Baum`. Als zentrales Beipiel für das Gitter sei das Eulersche Intervallgitter bzw. Tonnetz genannt. ${ }^{4}$

In vielen Abhandlungen zur Harmonielehre findet man ein zweidimensionales Gitter als geometrisches Modell für die Erfassung von Tonbeziehungen. Die Punkte dieses Gitters denotieren entweder die Oktavklassen von Intervallen (Intervallgitter) oder deren Diskanttöne (Tonnetz), die manchmal auch `Tonigkeiten genannt werden. In der nachstehenden Abbildung (folgende Seite) ist die horizontale Achse die Quintachse und die vertikale die Terzachse. Die eingetragenen Tonnamen stehen für die Diskanttöne dieser Intervalle von einem festen Basiston $C$ aus. Für viele Musiktheoretiker ist dieses Gitter verbunden mit der Auffassung von einem reinen Stimmungsprinzip in Oktaven, Quinten und Terzen. ${ }^{5}$

Das prominenteste Beispiel für ein Baum-Modell der Tonverwandtschaften ist die Notenschrift. Akzidentielle Töne werden dabei als von Stammtönen abgeleitet angesehen. Die dabei entstehende Hierarchie ist unverzweigt, wenn man berücksichtigt, daß Konstellationen wie \#b oder b\# nicht in Frage kommen. Verzweigte Hierarchien tre-

3 Die Tonapperzeption entspricht im Grundgedanken Mazzolas Diskantabbildung, die jedem Basiston in Abhängigkeit von einem Tonvektor einen Diskant zuordnet. Der Unterschied besteht lediglich in der Wahl des Raumes, in der diese Zuordnung stattfindet.

4 Es ist wichtig, zwischen sIntervallgitter und `Tonnetz streng zu unterscheiden, denn in der oftmals anzutreffenden Nachlässigkeit schlägt sich gerade die Vermischung von Tonvektoren und Intervallen als Zweitonmengen nieder.

5 Bei jener Auffassung der Gitterpunkte als Oktavklassen von ideell rein-gestimmten Tönen werden die arithmetischen Beziehungen von sFrequenzen $r=2^{o} \cdot 3^{q} \cdot 5^{t}$ unterscheiden. Fixiert man eine Ausgangsfrequenz $f_{0}$, so entspricht jeder auf $f_{0}$ bezogenen Frequenz $f=f_{0} r=f_{0} \cdot 2^{o} \cdot 3^{q} \cdot 5^{t}$ der Gitterpunkt mit den Koordinaten (q, t), wobei q die Quintkoordinate und t die Terzkoordinate bezeichnet. Unter Einbeziehung weiterer Primfaktoren in die Nenner und Zähler der Frequenzen kommen beim Tongitter weitere Dimensionen ins Spiel. Autoren wie Sigfrid Karg-Elert und auch Martin Vogel beziehen eine Septimenkoordinate in ihre Tonsysteme ein. 


\begin{tabular}{lll|lllll} 
& & & & & & & \\
\hline$G$ & D & A & E & B & F\# & C\# & G\# \\
\hline $\mathrm{Eb}$ & $\mathrm{Bb}$ & $\mathrm{F}$ & $\mathrm{C}$ & $\mathrm{G}$ & $\mathrm{D}$ & $\mathrm{A}$ & $\mathrm{E}$ \\
\hline $\mathrm{Cb}$ & $\mathrm{Gb}$ & $\mathrm{Db}$ & $\mathrm{Ab}$ & $\mathrm{Eb}$ & $\mathrm{Bb}$ & $\mathrm{Fb}$ & $\mathrm{C}$
\end{tabular}

ten jedoch auf, wenn man die Möglichkeit einräumt, daß akzidentielle Töne ihrerseits Verwandtschaftsbeziehungen zu stammtonartig abgeleiteten Tönen aufbauen. Ein anderes Beispiel für hierarchisch verzweigte Tonbeziehungen sind die sogenannten `Klammerfunktionen in der Funktionsharmonik: z.B. Dominante der Subdominantparallele, Doppel- oder Wechseldominante, Subdominante der Mollparallele, usw. Ein recht kompliziertes System der hierarchischen Ableitung von Tonbeziehungen wird in der Tradition nach Heinrich Schenker untersucht. Allerdings handelt es sich dabei vor allem um syntagmatische Tonbeziehungen, die sich deshalb nicht unmittelbar auf die vorliegende Untersuchung beziehen lassen.

Wie verhalten sich diese beiden Auffassungen vom Gitter und vom hierarchisch verzweigten Baum zueinander? Wir nähern uns dem Problem durch eine Charakterisierung der ıIntensionen scher Tonbeziehungen. Es geht im folgenden nicht(!) um die begriffliche Intension bzw. Extension des musiktheoretischen Begriffs »Tonverwandtschaft«, sondern wir meinen eine Modellierung der genuin ımusikalischen Intension bzw. Extension dessen, was wir im Rahmen dieses musiktheoretischen Ansatzes unter einzelnen Tonverwandtschaften verstehen wollen. Die folgenden drei Punkte geben einen ersten Grundriß für unseren Ansatz:

- Intension: Es sei ein Repertoire von sapperzipierbaren sind ausgewählte Intervalle, die zur Stiftung von Tonverwandtschaft herangezogen werden können. Sequenzen solcher Intervalle sollen als >Apperzeptionspläne ‘ bezeichnet werden. Es handelt sich um Pläne für verkettete Tonverwandtschaft stiftende Operationen. Jeder Tonverwandtschaft entspricht als Intension eine geeignete Menge solcher Apperzeptionspläne.

- Extension: Es sei ein Repertoire von Tonorten gegeben. Für einen fest gewählten Ausgangstonort entspricht jeder Tonverwandtschaft als Extension eine Menge von Tonorten. Gemeint sind diejenigen Tonorte, die zum Ausgangston in dem besagten Tonverwandtschaftsverhältnis stehen.

- Apperzeption: Der Zusammenhang von Intension und Extension einer Tonverwandtschaft sollte durch eine Apperzeptionsvorschrift beschrieben werden. Sie ordnet bei gegebenem Ausgangstonort jedem Apperzeptionsplan aus der Intension einer Tonverwandtschaft einen Tonort aus ihrer Extension zu. 
Die einzelnen Pfade in hierarchisch verzweigten Baummodellen verkörpern als Apperzeptionspläne zweifellos die intensionale Komponente der Tonverwandtschaften. In unserer konkreten Auffassung dieser Pfade als Sequenzen von Tonvektoren beziehen wir uns auf Martin Vogels Begriff der >Apperzeptionsbahn`. Vogels Interval-Sigel dienen nicht nur zur Notation von Intervallen in ihrer additiven Zusammensetzung aus Quinten, Terzen und Septimen, sondern auch zur Erfassung der konkreten Reihenfolge von Apperzeptionsschritten. So läßt sich die große Sexte $A$ zum Basiston $C$ in zwei Schritten als Terz der Unterquinte $F$ (Interval-Sigel $-Q T$ ) oder aber als Unterquinte der Terz (Interval-Sigel $T-Q$ ) auffassen (siehe Vogel 1975, 117).

Allerdings müssen wir den Terminus der »Apperzeptionsbahn« durch »Apperzeptionsplan« ersetzen, weil bei Vogel das Gitter in doppelter Rolle auftritt. Als Tonnetz modelliert es zugleich die Extensionen der Tonverwandtschaften. Martin Vogel und auch andere Theoretiker mit einer gewissen Affinität zur Obertonlehre setzen den Raum der durch Apperzeption erreichbaren Tonorte mit den Diskanttönen gleich, die man durch die sukzessive Addition der apperzipierbaren Intervalle von einem Basiston aus erreicht. Übersetzt man jene Basis- und Diskanttöne in idealisierte Frequenzen $f$, so steht hinter jener Auffassung ein Apperzeptionsprinzip, welches einer Übertragung des Fechnerschen Gesetzes aus dem Bereich der Psychoakustik in die Musiktheorie gleichkommt.

Diese Sichtweise ist recht aufschlußreich, weil die Funktionalgleichung der Exponentialfunktion $f \cdot \exp \left(v_{1}+v_{2}\right)=f \cdot \exp \left(v_{1}\right) \cdot \exp \left(v_{2}\right)$ die additiven Verknüpfungen $v_{1}+v_{2}$ innerhalb der Intension mit jener des sukzessiven Apperzipierens $f \mapsto f \cdot \exp \left(v_{1}\right) \mapsto f \cdot \exp \left(v_{1}\right)$ verbindet.

Aufgrund der Vertauschbarkeit der Apperzeptionsreihenfolge, die sich als Konsequenz aus diesem eindimensionalen Prinzip ergibt, ist klar, daß sich die hierarchische Verzweigung von Apperzeptionsplänen nicht in den zugehörigen Extensionen niederschlagen kann. Hierin mag einer der Hauptgründe liegen, warum gerade diejenigen Theoretiker, denen die Verzweigung der Tonverwandtschaften am Herzen liegt, das Tonnetz als Modell für deren Extensionen meiden. Aber selbst das Motto »Der Weg ist das Ziel« sollte nicht ein Desinteresse an Wegekreuzungen implizieren. Das Baummodell unterscheidet sich vom Gitter ja gerade darin, daß es Kreuzungen systematisch ausschließt. Es spricht einiges dagegen, die Tonorte - d.h. die Extensionen der Apperzeptionspläne - als die äußersten Zweiglein eines riesigen Baumes anzusehen. Viel näher liegt da die Vorstellung von einem Tonnetz, entlang dessen Netzlinien die durch die Apperzeptionspläne definierten Apperzeptionsbahnen verlaufen. Es spricht allerdings auch einiges dagegen, daß das traditionelle Tonnetz bereits ein geeignetes Modell dazu ist. Einer der Gründe liegt in der bereits erwähnten Unsensibilität gegenüber jeglicher Vertauschung in der Apperzeptionsreihenfolge. Ein weiterer Grund sind die sogenannten »Kommata».

\subsection{Ein `Kommar zwischen den Schulen}

Wenn man allein das traditionelle Tonnetz zum Ausgangspunkt des Studiums von Tonbeziehungen macht, ist man mit einem bekannten Problem konfrontiert: Alle Gitterpunkte mit dem Abstandsintervall $(4,-1)$ ( = 4 Quinten - 1 Terz) werden in der Notenschrift, die man für eine Theorie des Tondenkens durchaus ernst nehmen muß, nicht 
voneinander unterschieden. Die Tatsache, daß es zu jedem Gitterpunkt des Tonnetzes einen homonymen Punkt auf der Quintachse gibt, könnte man zum Anlaß nehmen, die Zweidimensionalität des Gitters aufzugeben.

Jenes syntonische Abstandsintervall trennt nicht nur die »pythagoräische« von der »reinen« Terz, sondern auch zwei musiktheoretische Traditionen: die Stufenlehre und die Funktionstheorie.

- Die Stufenlehre gründete sich unmittelbar auf die (eindimensionale) diatonische Achse und blieb dabei sehr nah mit der Notenschrift verbunden. Damit handelte sie sich systematisch das Problem ein, akzidentiellen Tönen auch nur »akzidentiell « Aufmerksamkeit widmen zu können, nämlich entlang hierarchisch verzweigter Bäume, die keine »Wegekreuzungen« kennen. Wenn es in diesem Ansatz eine extensionale Komponente gibt, dann ist es die der Noten. Dort verschwindet das syntonische Komma ebenso wie die Unterscheidung zwischen vorzeichenbehafteten Stammtönen und akzidentiellen Tönen

- Die Funktionstheorie stellte die Terzverwandtschaft als wichtige paradigmatische Beziehung neben die Quintverwandtschaft und mußte damit kommaverschiedene Töne in Kauf nehmen, wie etwa die Subdominantsexte und die Dominantquinte. Bei allzu starker Fokussierung auf die Tonhöhe wurden dabei oft Harmonielehre und Intonationslehre normativ miteinander gekoppelt.

Die heutige Musiktheorie neigt eher dazu, jene Schulen als historisch gewachsene Lehrgebäude zu akzeptieren, ohne die wissenschaftliche Forderung nach einer einheitlichen Theorie zu stellen. Vertritt man jedoch den Anspruch, eine einheitliche Theorie zu schaffen, so kommt man nicht umhin, den Dissens zwischen »Komma-Ignoranten« und »Komma-Enthusiasten« als ein ungelöstes Problem der Musiktheorie zu deuten.

Zur Unterstreichnung dieser These führen wir Hugo Riemanns Standpunkt dazu an. Sein Beispiel der Dreiklangsfolge $C A d G$, bei der je von Akkord zu Akkord ein Ton liegenbleibt, charakterisiert er wie folgt:

„Die Kadenz bringt den Parallelklang der Subdominante $d f$ a mit Vorausschickung seiner Dominante und geht dann über die Dominante zur Tonika zurück. Schon M. Hauptmann hat die Behauptung aufgestellt, daß dieses $d$ fa [...] keine reine Harmonie sei, da ihre Quinte $d$ a um ein syntonisches Komma (= 1/10 Ganzton) zu klein $(=27: 40)$ sei. [...] Der Alt müßte also im zweiten Takt $\underline{d}$ in $d$ verwandeln ( $\underline{d}$ als Unterquinte der Terz der Subdominante, $d$ als Quinte der Dominante). Unsere musikalische Praxis weiß von diesem zweierlei $d$ in C-Dur nichts, und unser Tonbewußtsein weiß noch weniger etwas davon, daß das $d: f: \underline{a}$ kein reiner Mollakkord, sondern eine Art verminderter Dreiklang (mit der Quinte 27:40 statt 2:3) wäre. Zweifellos stellen wir uns den d-Moll-Akkord als Parallelklang des F-Dur-Akkordes vor: und nicht als ein Mixtum compositum von Subdominante und Dominante, wie Hauptmann will: Wäre er nicht ein reiner Dreiklang, so könnten wir uns nicht seine Dominante vorstellen ... Mit anderen Worten: Unsere Vorstellung weiß nichts von der Stimmungsdifferenz von $\underline{d}$ und $d$, sondern setzt beide gleich, stellt $d$ als Unterquinte von $\underline{a}$ und doch zugleich auch als Oberquinte von $g$ vor. Diese enharmonische Identifikation der um das syntonische Komma verschiedenen akustischen Werte ist für unser Musikhören schlechterdings unentbehrlich." (Riemann 1914/15, 17) 
Riemann verbindet zwei Forderungen, deren Verträglichkeit nicht auf der Hand liegt. Einerseits postuliert er den reinen Molldreiklang und grenzt ihn von einem anderen Dreiklang ab, und gleichzeitig postuliert er die syntonische Identifikation. Würde man diese Identifikation als bloße mengentheoretische Klassenbildung schon in die Konstruktion des Tonsystems selbst einbeziehen, dann bliebe von der zweidimensionalen Gitterstruktur nur ein eindimensionaler `Faktorraum übrig, wie ihn auch die Stufenlehre implizit vorraussetzt. Doch dann wäre für die feine Unterscheidung des reinen Molldreiklangs von jenem anderen Dreiklang kein Beschreibungsrahmen mehr vorhanden. Riemann muß - aus unserer Sicht - mit der syntonischen Identifikation also einen unmerklichen Apperzeptionsakt meinen. Wir fassen Riemanns Forderungen in folgender Formulierung:

(1) Der syntonische Tonvektor ist nicht apperzipierbar.

(2) Die syntonischen Apperzeptionen sind dennoch möglich und können unmerklich vonstatten gehen.

\subsection{Tonorte und Enharmonizität}

Zu den offenen Fragen des Tonsystems gehört zweifellos auch das Problem, die enharmonische Identifikation zu erklären. Dazu findet man bei Hermann Pfrogner (1953), der zweifellos zu den Theoretikern gehört, die das Tonsystem essentiell als einen autonomen Gegenstandsbereich jenseits der Psychoakustik betrachten, folgende Auffassung:

»... Es ist eigentlich kaum glaublich und leider doch wahr, daß bis heute viele, wenn nicht die meisten Musiker, fasziniert von der gleichschwebenden Temperatur sals einer der genialsten Erfindungen des menschlichen Geistes`, völlig übersehen, daß z.B. eine Modulation von a- nach c-moll, vermittels des verminderten Septakkords, durch unsere Tastenstimmung nie und nimmer ursprünglich bedingt sein kann. Wenn nicht auf musikalischer Ebene der Tonwert gis sich in den Tonwert as verwandeln ließe oder umgekehrt - unsere Temperatur allein, von der empirischen Ebene aus, vermöchte einer solchen Modulation niemals musikalische Überzeugungskraft zu verleihen. Hinter dem Kompromiß der Tastenstimmung muß also noch etwas anderes stehen, etwas, das diesen Kompromiß überhaupt erst ermöglichte, sonst hätte man nämlich damit niemals Musik machen können. Die gleichschwebende Temperatur wäre dann ein Kuriosum geblieben, sie stände in einer Reihe neben dem seinerzeit aufsehenerregenden, heute längst vergessenen »enharmonischen« Archicembalo der Renaissancetheoretiker in einem verstaubten Raritätenkabinett, aber weder Bachs Wohltemperiertes Klavier noch Schönbergs Suite für Klavier, op. 25, hätten jemals das Licht der Welt erblickt.« (Pfrogner 1953, 28)

Wenn sich jene Verwandlung des Tonwerts gis in den Tonwert as als Apperzeption vollziehen soll, so ergibt sich daraus folgende Forderung für eine Theorie der Tonapperzeption:

Es muß einen Apperzeptionsplan geben, der in der Summe einen Tonvektor der dreifachen Terz ergibt und dessen Zieltonort mit dem der Prime übereinstimmt. Oder anders ausgedrückt: Jener gesuchte Apperzeptionsplan und die Prime (als trivialer Apperzeptionsplan) gehören beide zur Intension ein und deselben Tonortes. 
Pfrogners Begriffe des Tonwertes und des Tonortes lassen sich in enge Beziehung zu unseren Begriffen des Tonvektors und des Tonortes setzen. ${ }^{6}$

Abschließend wollen wir auch die Auffassung eines Praktikers zum Thema der enharmonischen Identifikation zur Kenntnis nehmen. In der von Richard Strauss ergänzten und revidierten Ausgabe der Instrumentationslehre von Hector Berlioz findet man folgende Anmerkung, die sich unter anderem auf eine Stelle in Glucks Orpheus bezieht, wo fis und ges sogar gleichzeitig erklingen.

Die alte Anmaßung der Akustiker, mit aller Gewalt das Resultat ihrer Berechnungen in die Praxis einer Kunst einzuführen, welche vor allem auf dem Studium der durch Töne auf das menschliche Ohr hervorgebrachten Eindrücke beruht, ist heutzutage nicht mehr haltbar. Soviel ist sicher, daß die Musik sie energisch zurückweist und überhaupt nur bestehen kann, wenn sie dieselbe zurückweist. Sicher ist ferner, daß die entgegengesetzten Modifikationen des Intervalls zwischen zwei Tönen, die sich anziehen, in der musikalischen Praxis sehr feine Nuancen sind, welche Virtuosen und Sänger mit größter Vorsicht anwenden, welche die Orchesterspieler im allgemeinen vermeiden und die von den Komponisten in Voraussicht ihrer Verwendung ganz besonders behandelt werden müssen. Ebenso sicher ist es endlich, daß die vorwiegende Mehrheit der Musiker sich ihrer im harmonischen Zusammenspiel instinktmäßig enthält. Daraus folgt, daß die von den Akustikern als miteinander unverträglich bezeichneten Töne sich in der musikalischen Praxis sehr gut miteinander vertragen, und daß die nach den Berechnungen als falsch erklärten Verhältnisse von dem Ohre als richtig empfunden werden; das Ohr läßt die unmerklichen Unterschiede, den Ansichten der Mathematiker zum Trotz, vollständig unbeachtet. (Berlioz/Strauss 1904, 429)

Wir stimmen Berlioz in seiner Argumentation voll und ganz zu. Dennoch wird unser Modell den Ansichten vieler Praktiker zum Trotz eine exakte mathematische Definition dessen geben, was ein sunmerklicher Unterschied` zweier Apperzeptionspläne hinsichtlich ihrer scheinbaren Zieltöne ist, und wir zeigen, daß es mittels dieser Definition gelingt, das Phänomen der enharmonischen Identifikation zu erklären.

\section{Ein geometrisches Modell}

Aus den Überlegungen des ersten Abschnitts ist klar geworden, daß das klassische, auf der eindimensionalen Exponentialfunktion beruhende Apperzeptionsprinzip nicht geeignet ist, den sonstigen theoretischen Anforderungen gerecht zu werden. Allein schon die Forderung nach einer Nichtvertauschbarkeit von Apperzeptionsschritten legt ein Modell nach der Art des nun vorgestellten nahe.

6 Im Zuge des weiteren Ausbaus des vorliegenden Theorieansatzes verspricht eine ausführliche Auseinandersetzung mit Pfrogners Auffassung eine Bereicherung der Argumentation. 


\subsection{Der Raum $\mathbb{T}$ der geometrischen Töne}

Es bezeichne $M_{2}(\mathbb{R})$ die 4-dimensionale $\mathbb{R}$-Algebra der $2 \times 2$-Matrizen mit reellen Einträgen,

$$
M_{2}(\mathbb{R})=\left\{\left(\begin{array}{ll}
a & b \\
c & d
\end{array}\right) \quad \mid \quad a, b, c, d \in \mathbb{R}\right\}
$$

mit den Operationen der Skalarmultiplikation, Matrixaddition und Matrixmultiplikation. Als Standardbasis betrachten wir die 4 Matrizen $\varepsilon_{1}, \varepsilon_{2}, \varepsilon_{3}, \varepsilon_{4}$ :

$$
\varepsilon_{1}=\left(\begin{array}{ll}
1 & 0 \\
0 & 0
\end{array}\right), \quad \varepsilon_{2}=\left(\begin{array}{ll}
0 & 1 \\
0 & 0
\end{array}\right), \quad \varepsilon_{3}=\left(\begin{array}{ll}
0 & 0 \\
1 & 0
\end{array}\right), \quad \varepsilon_{4}=\left(\begin{array}{ll}
0 & 0 \\
0 & 1
\end{array}\right)
$$

Bezüglich der Standardbasis sind die 4 Matrixeinträge $a, b, c$ und $d$ zugleich die Koordinaten des zugehörigen 4-Vektors. Daneben betrachten wir die `dia-syntonische Basis`, bestehend aus den Matrizen:

$$
\delta_{0}=\left(\begin{array}{ll}
1 & 0 \\
0 & 1
\end{array}\right), \quad \sigma_{0}=\left(\begin{array}{cc}
1 & 0 \\
0 & -1
\end{array}\right), \quad \sigma_{1}=\left(\begin{array}{ll}
0 & 1 \\
1 & 0
\end{array}\right), \quad \delta_{1}=\left(\begin{array}{cc}
0 & 1 \\
-1 & 0
\end{array}\right)
$$

Die von $\delta_{0}$ und $\delta_{1}$ aufgespannte Ebene nennen wir die sdiatonische Ebene nen die $\delta_{0}$-Achse als reelle lineare Isomorphismus $\Phi: M_{2}(\mathbb{R}) \rightarrow \mathbb{R}^{4}$,

$$
\Phi\left(\left(\begin{array}{ll}
a & b \\
c & d
\end{array}\right)\right)=\left(\frac{a+d}{2}, \frac{a-d}{2}, \frac{b+c}{2}, \frac{b-c}{2}\right)
$$

überführt die Basis $\left\{\delta_{0}, \sigma_{0}, \sigma_{1}, \delta_{1}\right\}$ in die kanonische Basis des $\mathbb{R}^{4}$,

$$
\begin{array}{ll}
\Phi\left(\delta_{0}\right)=(1,0,0,0), & \Phi\left(\sigma_{0}\right)=(0,1,0,0), \\
\Phi\left(\sigma_{1}\right)=(0,0,1,0), & \Phi\left(\delta_{1}\right)=(0,0,0,1) .
\end{array}
$$

7 Die von Martin Ebeling angestellten Untersuchungen lassen sich im Rahmen des vorgestellten vierdimensionalen Modells auf die diatonische Ebene beziehen, insofern diese Ebene via Addition und Multiplikation von $2 \times 2$-Matrizen mit der komplexen Zahlenebene identifiziert werden kann. Auch die Einschränkung der dia-syntonischen Norm stimmt mit der Norm (genauer: dem Normquadrat) komplexer Zahlen überein. Eine weiterführende Diskussion von Ebelings Ansatz im Lichte der vorliegenden Untersuchungen könnte sich bei der Suche nach einem Zusammenhang von Perzeption und Apperzeption als fruchtbar erweisen. 
Im Vektorraum $\mathbb{R}^{4}$ betrachten wir nun das (indefinite nicht-ausgeartete) Skalarprodukt $\langle.,\rangle:. \mathbb{R}^{4} \times \mathbb{R}^{4} \rightarrow \mathbb{R}^{4}$

$$
\left\langle\left(d_{0}, s_{0}, s_{1}, d_{1}\right),\left(e_{0}, t_{0}, t_{1}, e_{1}\right)\right\rangle:=d_{0} e_{0}-s_{0} t_{0}-s_{1} t_{1}+d_{1} e_{1} .
$$

Die Bezeichnung der Koordinaten soll hierbei an die Zuordnung zwischen dia-syntonischer Basis in $M_{2}(\mathbb{R})$ und kanonischer Basis in $\mathbb{R}^{4}$ erinnern.

Definition 1. Den mit dem oben definierten Skalarprodukt $\langle.,$.$\rangle ausgestatteten Vek-$ torraum $\mathbb{R}^{4}$ bezeichnen wir mit $\mathbb{T}=\mathbb{T}_{(+,-,-,)}^{4}$ und nennen ihn den $>$ Raum der geometrischen Tönes. Sein indefinites, nicht-ausgeartetes Skalarprodukt nennen wir die >diasyntonische Paarung`. Die davon induzierte pseudo-euklidische Norm $\|\|:. \mathbb{T} \rightarrow \mathbb{R}$ mit $\left\|\left(d_{0}, s_{0}, s_{1}, d_{1}\right)\right\|:=d_{0}^{2}-s_{0}^{2}-s_{1}^{2}+d_{1}^{2}$ heißt $>$ dia-syntonische Norm .

Ein geometrischer Ton $T \in \mathbb{T}$ heißt

1. diatonartig, wenn $\|T\|>0$ ist,

2. quintartig für $\|T\|=0$ und

3. syntonartig, wenn $\|T\|<0$ gilt.

Die Mengen der diatonartigen, der quintartigen bzw. der syntonartigen geometrischen Töne bezeichnen wir mit $\mathbb{T}^{+}, \mathbb{T}^{0}$ bzw. $\mathbb{T}^{-}$.

Als nächstes sollen die dia-syntonische Paarung und die dia-syntonischen Norm mit Hilfe des Isomorphismus $\Phi$ in den Raum $M_{2}(\mathbb{R})$ übertragen werden. Determinate und Spur einer Matrix seien wie üblich gegeben durch die Abbildungen det, $\operatorname{tr}: M_{2}(\mathbb{R}) \rightarrow \mathbb{R}$,

$$
\operatorname{det}\left(\begin{array}{ll}
a & b \\
c & d
\end{array}\right)=a d-b c, \quad \operatorname{tr}\left(\begin{array}{ll}
a & b \\
c & d
\end{array}\right)=a+d .
$$

Ferner sei die Abbildung $\sim: M_{2}(\mathbb{R}) \rightarrow M_{2}(\mathbb{R})$ gegeben durch die Vorschrift

$$
\left(\begin{array}{ll}
\widetilde{a} & b \\
c & d
\end{array}\right)=\left(\begin{array}{cc}
d & -b \\
-c & a
\end{array}\right)
$$

Dann definieren wir die Standardpaarung $\langle., .\rangle_{0}: M_{2}(\mathbb{R}) \times M_{2}(\mathbb{R}) \rightarrow \mathbb{R}$ für $2 \times 2$-Matrizen mit Hilfe der Matrixmultiplikation $\circ: M_{2}(\mathbb{R}) \times M_{2}(\mathbb{R}) \rightarrow M_{2}(\mathbb{R})$ durch die Vorschrift

$$
\langle\alpha, \beta\rangle_{0}:=\frac{1}{2} \operatorname{tr}(\alpha \circ \widetilde{\beta})
$$

sowie die Standardnorm $\|\cdot\|_{0}: M_{2}(\mathbb{R}) \rightarrow \mathbb{R}$ vermöge 


$$
\|\alpha\|_{0}:=\langle\alpha, \alpha\rangle_{0}=\frac{1}{2} \operatorname{tr}(\alpha \circ \tilde{\alpha})=\operatorname{det}(\alpha)
$$

Lemma 1 Der lineare Isomorphismus $\Phi: M_{2}(\mathbb{R}) \rightarrow \mathbb{T}_{(+,-,-,+)}^{4}$ ist eine Isometrie des Raumes $\left(M_{2}(\mathbb{R}),\langle., .\rangle_{0}\right)$ auf den Raum $\left(\mathbb{T}_{(+,-,-,+}^{4},\langle.,\rangle.\right)$, d.h. für alle $\alpha$ und $\beta$ in $M_{2}(\mathbb{R})$ gilt

$$
\langle\alpha, \beta\rangle_{0}=\langle\Phi(\alpha), \Phi(\beta)\rangle \quad \text { und } \quad\|\alpha\|_{0}=\|\Phi(\alpha)\|_{\text {. }}
$$

Beweis:

Seien $\alpha=\left(\begin{array}{ll}a_{1} & b_{1} \\ c_{1} & d_{1}\end{array}\right) \quad$ und $\beta=\left(\begin{array}{ll}a_{2} & b_{2} \\ c_{2} & d_{2}\end{array}\right)$, dann ist

$$
\begin{aligned}
\langle\alpha, \beta\rangle_{0}=\frac{1}{2} \operatorname{tr}(\alpha \cdot \widetilde{\beta}) & =\frac{1}{2} \operatorname{tr}\left(\begin{array}{cc}
a_{1} d_{2}-b_{1} c_{2} & -a_{1} b_{2}+b_{1} a_{2} \\
c_{1} d_{2}-d_{1} c_{2} & d_{1} a_{2}-c_{1} b_{2}
\end{array}\right) \\
& =\frac{1}{2}\left(a_{1} d_{2}+d_{1} a_{2}-b_{1} c_{2}-c_{1} b_{2}\right) .
\end{aligned}
$$

Andererseits ergibt sich

$$
\begin{aligned}
\langle\Phi(\alpha), \Phi(\beta)\rangle= & \frac{\left(a_{1}+d_{1}\right)\left(a_{2}+d_{2}\right)}{4}-\frac{\left(a_{1}-d_{1}\right)\left(a_{2}-d_{2}\right)}{4} \\
& -\frac{\left(b_{1}+c_{1}\right)\left(b_{2}+c_{2}\right)}{4}+\frac{\left(b_{1}-c_{1}\right)\left(b_{2}-c_{2}\right)}{4} \\
= & \frac{1}{2}\left(a_{1} d_{2}+d_{1} a_{2}-b_{1} c_{2}-c_{1} b_{2}\right) .
\end{aligned}
$$

\subsection{Tonorte und Tonvektoren}

Die geometrischen Töne fungieren als gemeinsamer Umgebungsraum für die `Tonorte` und `Tonvektoren`, die im Rahmen unserer Untersuchungen zwei grundlegend verschiedene Gegenstandstypen sind. Da unter Umständen ein und derselbe geometrische Ton sowohl als Tonort als auch als Tonvektor aufgefaßt werden kann, werden wir die betreffende Rolle stets kennzeichnen.

Definition 2. Unter Tonorten verstehen wir geometrische Töne $T \in \mathbb{T}$ der diasyntonischen Norm $\|T\|=1$. Die Tonorte sind also die Punkte auf der Quadrik $\mathbb{S}=\left\{T=\left(d_{0}, s_{0}, s_{1}, d_{1}\right) \mid\|T\|=d_{0}^{2}-s_{0}^{2}-s_{1}^{2}+d_{1}^{2}=1\right\}$.

Der dreidimensionale Raum $S$ der Tonorte ist mathematisch auf zwei Weisen ausgezeichnet: geometrisch als der dreidimensionale anti-de Sitter-Raum adS mit seiner Lorentz-Metrik konstanter Schnittkrümmung -1 und gruppentheoretisch als die Lie- 
Gruppe $S L(2, \mathbb{R})$ mit der Ad-invarianten Killingform, die eine bi-invariante pseudo-riemannsche Metrik definiert. Die Transformation $\Phi$ überführt die eine Sicht in die andere. Sie vermittelt eine isometrische Einbettung von $S L(2, \mathbb{R})$ als Hyperfläche in die flache pseudo-riemannsche Mannigfaltigkeit T. Die Geodätischen entsprechen gruppentheoretisch den Translaten von 1-Parameter-Untergruppen, d.h., im Einselement der Gruppe stimmen Gruppenexponential und Exponential der Lorentz-Mannigfaltigkeit überein.

Eine weitere - geometrische wie gruppentheoretische - Besonderheit des Raumes $\mathbb{S}$ ist die Tatsache, daß er ein kanonisches »Gitter «, d.h. eine diskrete Untergruppe enthält - die Gruppe $S L(2, \mathbb{Z})$, die sich musiktheoretisch als sehr bedeutsam erweist.

Definition 3. `Verallgemeinerte Tonorte nennen wir die diatonartigen geometrischen Töne, d.h. geometrische Töne mit positiver dia-syntonische Norm. Die Quadratwurzel dieser Norm nennen wir die `Frequenzkoordinate des verallgemeinerten Tonortes $T \in \mathbb{T}$. Den Raum aller verallgemeinerten Tonorte bezeichnen wir mit $\mathbb{G}$,

$$
\mathbb{G}=\{T \in \mathbb{T} \mid\|T\|>0\} .
$$

Die Abbildungen Freq $: \mathbb{G} \rightarrow \mathbb{R}$ und $R: \mathbb{G} \rightarrow \mathbb{S}$, Freq $(T):=\|T\|^{\frac{1}{2}}$ und $R(T):=\|T\|^{-\frac{1}{2}} \cdot T$, heißen `Frequenzabbildung bzw. `Tonortretraktion`. Letztere weist jedem verallgemeinerten Tonort einen (speziellen) Tonort in $\mathbb{S}$ derart zu, daß dabei jedes $T \in \mathbb{S}$ fest bleibt, $R(T)=T$.

Definition 4. `Tonvektoren sind geometrische Töne, deren erste Koordinate verschwindet, d.h. die Punkte der Hyperebene

$$
\mathbb{V}_{(-,-,+)}^{3}=\mathbb{V}=\left\{v=\left(d_{0}, s_{0}, s_{1}, d_{1}\right) \mid d_{0}=0\right\} .
$$

In Übertragung unserer Klassifikation der geometrischen Töne bezeichnen wir die Mengen der diatonartigen, der quintartigen bzw. der syntonartigen Tonvektoren mit $\mathbb{V}^{+}, \mathbb{V}^{0}$, bzw. $\mathbb{V}^{-}$.

Definition 5. Als sverallgemeinerte Tonvektoren betrachten wir beliebige geometrische Töne $v=\left(d_{0}, s_{0}, s_{1}, d_{1}\right) \in \mathbb{T}$.

Die Abbildung $h: \mathbb{T} \rightarrow \mathbb{R}$ mit $h(v)=h\left(\left(d_{0}, s_{0}, s_{1}, d_{1}\right)\right):=d_{0}$ heißt Höhenabbildung. Die Abbildung $r: \mathbb{T} \rightarrow \mathbb{V}, r(v):=v-\left(d_{0}, 0,0,0\right)$ heißt Tonvektorretraktion. Sie weist jedem verallgemeinerten Tonvektor einen (speziellen) Tonvektor aus $\mathbb{V}$ zu und läßt jedes $v \in \mathbb{V}$ fest, $r(v)=v$.

Unter der Isometrie $\Phi^{-1}$ geht die Hyperebene $\mathbb{V}$ in

$$
s l_{2}(\mathbb{R})=\left\{\alpha \in M_{2}(\mathbb{R}) \mid \operatorname{tr}(\alpha)=0\right\}
$$

über, und die Tonvektorretraktion bekommt die Gestalt

$$
r_{0}: M_{2}(\mathbb{R}) \rightarrow s l_{2}(\mathbb{R}), \quad r_{0}(\alpha)=\alpha-\frac{\operatorname{tr}(\alpha)}{2} \delta_{0} .
$$


Für den Tonort, der dem geometrischen Ton $(1,0,0,0) \in \mathbb{T}$ entspricht, führen wir die Bezeichnung $Z$ ein und nennen ihn den zzentralen Tonort. Die Bedingung für einen Tonvektor $v \in \mathbb{T}, z u \mathbb{V}$ zu gehören, ist gleichbedeutend damit, daß seine dia-syntonische Paarung mit $Z$ verschwindet, $\langle Z, v\rangle=0 . \mathbb{V}$ ist also die Tangentialhyperebene im Punkt $Z$ an den Raum $\mathbb{S}$ der Tonorte. Mit $\Phi$ nach $M_{2}(\mathbb{R})$ übertragen ist also $s l_{2}(\mathbb{R})$ die Tangentialhyperebene in $\delta_{0}$ an die Quadrik $S L_{2}(\mathbb{R})$.

In Ergänzung zu den bislang eingeführten Tonvektoren, mit denen wir immer sTonvektoren am zentralen Tonort meinen, betrachten wir nun auch für jeden anderen Tonort $T \in \mathbb{S}$ die `Tonvektoren am Tonort $T$, d.h. die Vektoren im Tangentialraum an die Hyperfläche $\mathbb{V}$ im Punkt $T$.

$U m$ für einen gegebenen Tonort $T \in \mathbb{S}$ den entsprechenden Tangentialraum $\mathbb{V}_{T}$ zu bestimmen, verwenden wir die Beschreibung dieser Hyperfläche als Gruppe reeller Matrizen der Determinante $1, \Phi^{-1}(\mathbb{S})=S L_{2}(\mathbb{R})$.

Der Tangentialraum dieser Lie-Gruppe im neutralen Element $\delta_{0}, s l_{2}(\mathbb{R})=\Phi^{-1}(\mathbb{V})$, ist die zugehörige Lie-Algebra, die Menge der reellen $2 \times 2$-Matrizen der Spur 0 .

Seien $\Phi^{-1}(T)=\left(\begin{array}{ll}A & B \\ C & D\end{array}\right), A D-B C=1$, ein fester Tonort und $\Phi^{-1}(v)=\left(\begin{array}{cc}a & b \\ C & -a\end{array}\right)$ ein variabler Tonvektor. Dann ist

$$
\Phi^{-1}(T) \circ \Phi^{-1}(v)=\left(\begin{array}{ll}
A a+B C & A b-B a \\
C a+D c & C b-D a
\end{array}\right)
$$

die »Verschiebung« (präziser: Linkstranslation) von $v$ in den Tonort $T$ in dieser LieGruppe. Für die weiteren Überlegungen erweist sich die Tatsache als entscheidend, daß die Einschränkung der Standardpaarung von $M_{2}(\mathbb{R})$ auf die Hyperfläche $S L_{2}(\mathbb{R})$ mit (einem Vielfachen) der durch die Killingform der Lie-Algebra $s l_{2}(\mathbb{R})$ definierten bi-invarianten Lorentz-Metrik zusammenfällt. Die Bi-invarianz der Metrik besagt gerade, daß die Paarung zweier verschobener Tonvektoren mit der der Ausgangstonvektoren übereinstimmt,

$$
\left\langle\Phi^{-1}(T) \circ \Phi^{-1}\left(v_{1}\right), \Phi^{-1}(T) \circ \Phi^{-1}\left(v_{2}\right)\right\rangle_{0}=\left\langle\Phi^{-1}\left(v_{1}\right), \Phi^{-1}\left(v_{2}\right)\right\rangle_{0} .
$$

Inbesondere bleibt damit die Eigenschaft eines Tonvektors, diatonartig, quintartig oder syntonartig zu sein, bei Verschiebung erhalten. Wir benutzen die Symbole $\mathbb{V}_{T}^{+}, \mathbb{V}_{T}^{0}$, bzw. $\mathbb{V}_{T}^{-}$zur Bezeichnung der Verschiebungen von $\mathbb{V}^{+}, \mathbb{V}^{0}$, bzw. $\mathbb{V}^{-}$in den Tonort $T$.

\subsection{Die Apperzeptionsformel}

Das klassische Apperzeptionsprinzip soll nun buchstäblich von idealisierten Frequenzen und Intervallen auf Tonorte und Tonvektoren übertragen werden. Dazu verwenden wir die Exponentialabbildung für reelle $2 \times 2$-Matrizen, 


$$
\begin{gathered}
\operatorname{Exp}: M_{2}(\mathbb{R}) \rightarrow M_{2}(\mathbb{R}), \\
\operatorname{Exp}(\alpha):=\sum_{k=0}^{\infty} \frac{1}{k !} \alpha^{k} .
\end{gathered}
$$

Wir fassen hier die Urbilder im Sinne von verallgemeinerten Tonvektoren auf und verstehen ihre Bilder unter der Exponentialfunktion als verallgemeinerte Tonorte. Aufgrund $\operatorname{der}$ Beziehung $\operatorname{det}(\operatorname{Exp}(\alpha)=\exp (\operatorname{tr}(\alpha)))$ sind dann die Bilder von (speziellen) Tonvektoren auch (spezielle) Tonorte, d.h.

$$
\left.\operatorname{Exp}\right|_{s l_{2}(\mathbb{R})}: s l_{2}(\mathbb{R}) \rightarrow S L_{2}(\mathbb{R}) .
$$

Das folgende Lemma enthält explizite Formeln für die Bilder dieser Exponentialfunktion, die zugleich eine geometrische Vorstellung von dieser Abbildung vermitteln sollen.

Lemma 2 Sei $\alpha \in s I_{2}(\mathbb{R})$ und $I=\sqrt{|\operatorname{det}(\alpha)|}$. Dann gilt

$$
\operatorname{Exp}(\alpha)=\left\{\begin{array}{cc}
\cos (I) \cdot \delta_{0}+\frac{\sin (I)}{I} \cdot \alpha & \operatorname{det}(\alpha)>0, \text { d.h. } \alpha \text { diaton }- \text { artig } \\
\delta_{0}+\alpha & \operatorname{det}(\alpha)=0, \text { d.h. } \alpha \text { quint }- \text { artig } \\
\cosh (I) \cdot \delta_{0}+\frac{\sinh (I)}{I} \cdot \alpha & \operatorname{det}(\alpha)<0, \text { d.h. } \alpha \text { synton }- \text { artig. }
\end{array}\right.
$$

In jedem der drei Fälle liegt das Exponential von $\alpha$ im Durchschnitt von $S L_{2}(\mathbb{R})$ mit der von $\delta_{0}$ und $\alpha$ aufgespannten Ebene in $M_{2}(\mathbb{R})$. Faßt man $\delta_{0}$ und $\operatorname{Exp}(\alpha)$ als Tonorte und $\alpha$ als Tonvektor auf, so gelangt man von $\delta_{0}=Z$ zu $\operatorname{Exp}(\alpha)$ entlang einer Geraden in Richtung von $\alpha$, die durch ein geeignetes Vielfaches des Zentraltons führt, d.h. durch einen verallgemeinerten Ton in $\delta_{0}$ - Richtung. Im Falle eines quintartigen Vektors $\alpha$ ist das Exponential eine einfache Addition aus Zentralton $Z$ und Argument $\alpha$ - die 1-Parameter-Untergruppe in dieser Richtung ist die zugehörige Gerade des Umgebungsraumes.

Bemerkung 1. Die enge Verbindung von Lorentz-Geometrie und Gruppenstruktur in $S L_{2}(\mathbb{R})$ findet ihren besonderen Ausdruck in der Tatsache, daß die Geodätischen (»verallgemeinerten Geraden«) dieses Raumes nichts anderes als die Translate der 1-ParameterUntergruppen sind. Diese Besonderheit wird im weiteren vielfach verwendet werden. Das obige Lemma besagt zum Beispiel, daß die Geodätischen in quintartigen Richtungen stets die zugehörigen Geraden im Umgebungsraum $M_{2}(\mathbb{R})$ sind. Allgemein enstehen die Geodätischen in $S L_{2}(\mathbb{R})$ als Durchschnitte von Hyperebenen im Umgebungsraum mit der Hyperfläche. Im Zentralton erhält man so für $I>0$ Hyperbeln und im Fall $I<0$ Ellipsen.

Wir verwenden dasselbe Symbol $\operatorname{Exp}: \mathbb{T} \rightarrow \mathbb{G}$, wenn wir mit Hilfe von $\Phi$ die Exponentialfunktion auf geometrische Töne $T \in \mathbb{T}$ anwenden, 


$$
\begin{gathered}
\operatorname{Exp}_{\mathbb{V}}: \mathbb{V} \rightarrow \mathbb{S}, \\
\operatorname{Exp}_{\mathbb{V}}(T)=\Phi\left(\operatorname{Exp}\left(\Phi^{-1}(T)\right)\right) .
\end{gathered}
$$

Desgleichen übertragen wir zur Vereinfachung der Notation die Multiplikation von Matrizen nach $\mathbb{T}$ :

$$
T_{1} \cdot T_{2}:=\Phi\left(\Phi^{-1}\left(T_{1}\right) \circ \Phi^{-1}\left(T_{2}\right)\right)
$$

Im folgenden Diagramm sind die verschiedenen Räume und Abbildungen dargestellt. Dabei bezeichnen $i_{S L_{2}(\mathbb{R})}, i_{s l_{2}(\mathbb{R})}$ und $j_{\mathbb{V}}, j_{\mathbb{S}}$ die Inklusion der jeweiligen Teilmenge in den Umgebungsraum $M_{2}(\mathbb{R})$ bzw. $\mathbb{T}$.

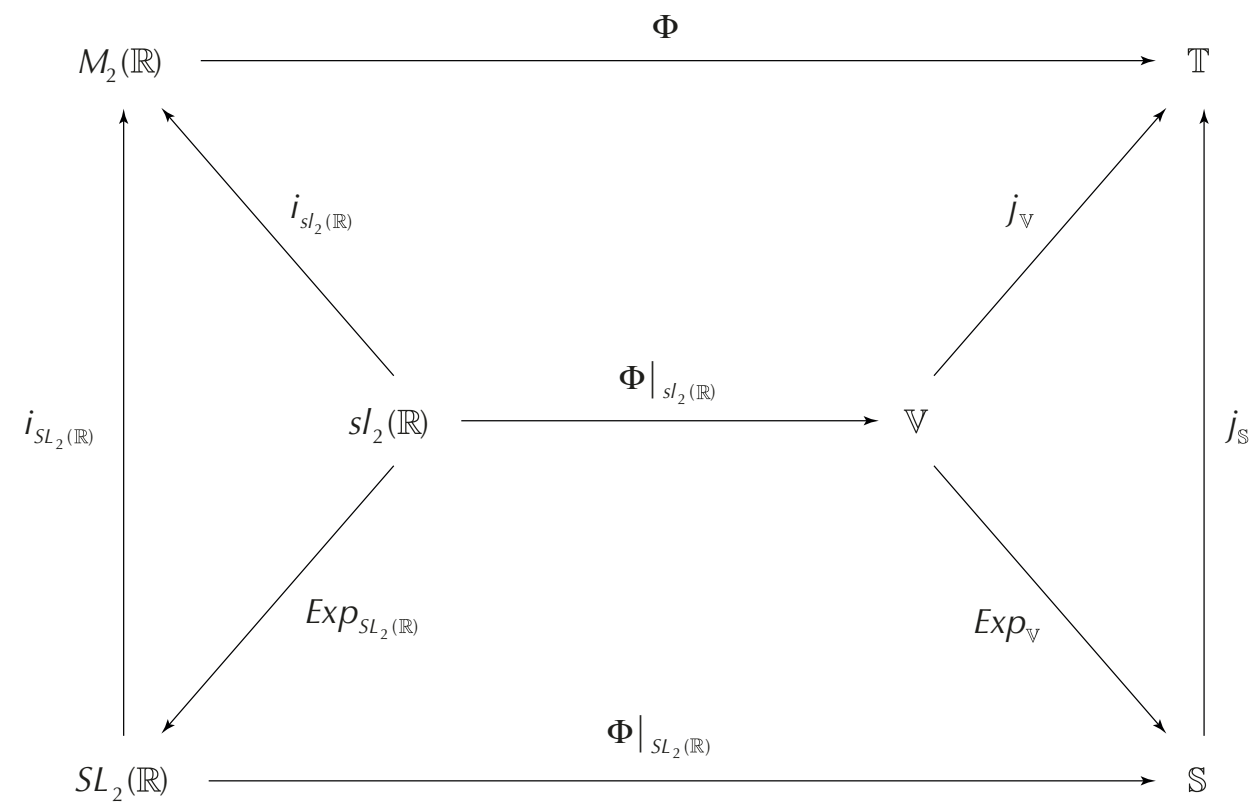

Definition 6. Die `Apperzeptionsabbildung App: $\mathbb{S} \times \mathbb{V} \rightarrow \mathbb{S}$ ist durch die folgende Vorschrift gegeben:

$$
\operatorname{App}(T, v):=T \circ \operatorname{Exp}_{\mathbb{v}}(v)
$$

$\operatorname{App}(T, v)$ heißt das ^Apperzeptionsziek des Tonvektors $v \in \mathbb{V}$ vom Tonort $T \in \mathbb{S}$ aus. Je nachdem, ob $v$ diatonartig, quintartig oder syntonartig ist, sprechen wir auch von einer diatonartigen, quintartigen bzw. syntonartigen Apperzeption. 
Im Abschnitt 3 postulieren wir aufgrund einer musiktheoretischen Überlegung, daß die Apperzeption stets quintartig oder diatonartig ist, d.h. wir postulieren, die Nichtapperzipierbarkeit syntonartiger Tonvektoren. Für jeden Tonort $T \in \mathbb{T}$ wird die Vereinigung $\mathbb{V}_{T}^{+} \cup \mathbb{V}_{T}^{0}$ deshalb auch sApperzeptionskegek in $T$ genannt. Jeder Apperzeptionskegel zerfällt nach Herausnahme des Nullvektors in zwei disjunkte Teilkegel, die im Fall des zentralen Tonorts $T=Z$ durch das Vorzeichen der letzten Koordinate $d_{1}$ bestimmt sind. Wir setzen

$$
\mathbb{V}^{\#}=\left\{v=\left(0, s_{0}, s_{1}, d_{1}\right) \in \mathbb{V} \mid \quad d_{1}>0,\|v\| \geq 0\right\}
$$

und

$$
\mathbb{V}^{b}=\left\{v=\left(0, s_{0}, s_{1}, d_{1}\right) \in \mathbb{V} \mid \quad d_{1}<0,\|v\| \geq 0\right\}
$$

$\mathbb{V}^{\#}$ heißt der \# >-Kegel und $\mathbb{V}^{b}$ der b >-Kegel . Für jeden vom Zentralton $Z$ verschiedenen Tonort $T \in \mathbb{T}$ entstehen die entsprechenden Kegel durch Verschiebung, $\mathbb{V}_{T}^{\#}:=T \cdot \mathbb{V}^{\#}$ und $\mathbb{V}_{T}^{b}:=T \cdot \mathbb{V}^{b}$.

Bemerkung 2. Die Apperzeptionsabbildung kann nach derselben Formel $\operatorname{APP}(T, v):=T \cdot \operatorname{Exp}(v)$ auch auf verallgemeinerte Tonorte $T$ und verallgemeinerte Tonvektoren $v$ angewandt werden. Unter Berücksichtigung der eindimensionalen Apperzeptionformel $\operatorname{app}(f, h):=f e^{h}$ für Frequenzen und Höhen ergibt sich aus der Zerlegung $v=h(v) \cdot \delta_{0}+r(v)$ und der Vertauschbarkeit der skalaren Matrix $h(v) \cdot \delta_{0}$ mit jeder anderen die folgende Beziehung:

$$
\begin{aligned}
\operatorname{APP}(T, v) & =\operatorname{APP}\left(\operatorname{Freq}(T) \cdot R(T), h(v) \cdot \delta_{0}+r(v)\right) \\
& =(\operatorname{Freq}(T) \cdot R(T)) \circ \operatorname{Exp}\left(h(v) \cdot \delta_{0}+r(v)\right) \\
& =\left(\operatorname{Freq}(T) \mathrm{e}^{h(v)}\right) \cdot(R(T) \circ \operatorname{Exp}(r(v))) \\
& =\operatorname{app}(\operatorname{Freq}(T), h(v)) \cdot \operatorname{App}(R(T), r(v)) .
\end{aligned}
$$

Diese Rechnung besagt, daß die (verallgemeinerte) Apperzeption, $A P P(T, v)$ (verallgemeinerter Tonvektoren $v$ von verallgemeinerten Tonorten $T$ aus), im Rahmen der Multiplikation von $2 \times 2$-Matrizen zerlegt werden kann in

- die eindimensionale Apperzeption app $(\operatorname{Freq}(T), h(v))$ der Höhe $h(v)$ von der Frequenz Freq $(T)$ aus und

- die Apperzeption $A p p(R(T), r(v))$ des Tonvektorretrakts $r(v)$ vom Tonortretrakt $R(T)$ aus.

Beide Teilapperzeptionen werden also in einem gemeinsamen Umgebungsraum beschrieben, erweisen sich dort jedoch als unabhängig voneinander. Für die vorliegende Untersuchung dient das als Legitimation, die verallgemeinerte Apperzeption nicht eingehender zu betrachten. Zugleich bietet sich aber die spätere Anknüpfung an die bereits erwähnten Untersuchungen Martin Ebelings unmittelbar an. 


\subsection{Apperzeptionspläne und Apperzeptionsbahnen}

Definition 7. Eine Sequenz $P=\left(v_{1}, \ldots, v_{k}\right) \in \mathbb{V}^{k}$ von Tonvektoren heißt $>$ Apperzeptionsplan Zieltonvektor dieses Apperzeptionsplans. Jeder Apperzeptionsplan induziert eine Abbildung $B[P]: \mathbb{S} \rightarrow \mathbb{S}^{k+1}$. Sie ordnet jedem Tonort T die Tonort-Sequenz der sukzessiven Apperzeptionen der Tonvektoren des Apperzeptionsplans zu:

$$
B[P](T):=\left(T, A p p\left(T, v_{1}\right), \ldots, A p p\left(\ldots A p p\left(A p p\left(T, v_{1}\right), v_{2}\right), \ldots, v_{k}\right) .\right.
$$

Für jeden festen Ausgangstonort $T$ heißt $B[P](T)$ die >Apperzeptionsbahn` zum Apperzeptionsplan $P$ vom Tonort $T$ aus. Den jeweils letzten Tonort $\Omega[P](T)$ in der Sequenz $B[P](T)$ nennen wir den `Zieltonort der durch $P$ von $T$ aus erzeugten Apperzeptionsbahn.

Apperzeptionsbahnen der Länge 1, die also aus der Apperzeption genau eines Tonvektors bestehen, heißen `Apperzeptionsschritte

Definition 8. Zwei Apperzeptionspläne $P_{1}$ und $P_{2}$ heißen szielgleichı, wenn ihre Zieltonorte vom zentralen Tonort $Z$ aus übereinstimmen, d.h., wenn $\Omega\left[P_{1}\right](Z)=\Omega\left[P_{2}\right](Z)$ gilt. Zwei Apperzeptionspläne $P_{1}$ und $P_{2}$ heißen sscheinbar zielgleichı, wenn ihre scheinbaren Zieltonvektoren übereinstimmen, wenn also $\Sigma\left(P_{1}\right)=\Sigma\left(P_{2}\right)$ ist. Zwei Apperzeptionspläne $P_{1}$ und $P_{2}$, die zielgleich sind, aber nicht scheinbar zielgleich, heißen hinsichtlich ihrer scheinbaren Zieltonvektoren ıunmerklich verschiedenء.

Bemerkung 3. Die Gruppenstruktur der Tonorte garantiert, daß aus der Zielgleichheit von Apperzeptionsplänen am zentralen Tonort die Zielgleichheit an jedem Tonort folgt.

Einen Sonderfall bilden ızyklische`Apperzeptionspläne, deren scheinbarer Zieltonvektor der Null- oder Primvektor ist. Wir nennen sie auch ıscheinbar trivialk.

Beispiel 1. Eine Apperzeptionsbahn, die zielgleich mit einem einzigen Apperzeptionsschritt ist, nennen wir ıschritthaftı. Nach Lemma 3 gibt es nur eine Situation, in der unterschiedliche Einzelschritte vom zentralen Tonort aus zum selben Zieltonort führen, nämlich alle Apperzeptionen $(r+2 \pi k) \delta_{1}, k \in \mathbb{Z}$ für festes $r \in \mathbb{R}$. Deshalb ist es zulässig, den Typ des Einzelschrittes, der einen schritthaften Apperzeptionsplan ersetzen kann, auf diesen zu übertragen: diatonartig, quintartig oder syntonartig.

Eine besondere Rolle spielen in unseren Untersuchungen Apperzeptionspläne, die aus einem Repertoire von nur vier quintartigen Tonvektoren gebildet werden. Sie entsprechen den geometrischen Tönen $\varepsilon_{2}$ und $\varepsilon_{3}$ aus der Standardbasis, sowie ihren Umkehrungen $-\varepsilon_{2}$ und $-\varepsilon_{3}$ :

- die `Quinte $\triangleleft=\left(0,0, \frac{1}{2}, \frac{1}{2}\right)$,

- die $>$ Quarte $\triangleleft-q=\left(0,0,-\frac{1}{2},-\frac{1}{2}\right)$,

- die >Leitquarte $>p=\left(0,0, \frac{1}{2},-\frac{1}{2}\right)$,

- die >Leitquarte $<-p=\left(0,0,-\frac{1}{2}, \frac{1}{2}\right)$.

Für die zugehörigen Apperzeptionen führen wir Kurzschreibweisen ein: 


$$
\begin{array}{ll}
T^{\triangleright}:=A p p(T, q)=T+q & T^{\triangleleft}:=\operatorname{App}(T,-q)=T-q \\
T^{\nabla}:=A p p(T, p)=T+p & T^{\triangleleft}:=A p p(T,-p)=T-p
\end{array}
$$

\section{Antworten im Modell}

Die nun folgenden Untersuchungen sind der Behandlung jener Probleme gewidmet, die im Eingangsabschnitt als bislang offene Fragen zum Tonsystem charakterisiert worden sind. Dabei wird es in verschiedenen Situationen immer wieder um die Frage gehen, ob gegebene Apperzeptionspläne zielgleich bzw. scheinbar zielgleich sind oder nicht.

Zunächst geht es um quintartige Apperzeptionspläne $P=\left(v_{1}, \ldots, v_{k}\right)$, die ausschließlich aus Tonvektoren $v_{i} \in\{q,-q, p,-p\}$ gebildet sind. Dabei ist es erforderlich, die ausgezeichnete Rolle, die jenen Tonvektoren zukommt, zu motivieren.

\subsection{Die Leitquarte}

Es ist wichtig, an dieser Stelle zu betonen, daß die Wahl der Basistonvektoren allein schon aus mathematischer Sicht denselben eine exponierte Rolle zuweist. Die musiktheoretische Diskussion dieser Auswahl ist also ein zentraler Punkt in der Weiterentwicklung des vorgestellten Theorieansatzes. Mit den nachfolgenden knappen Überlegungen soll zumindest die Plausibilität unserer Wahl belegt werden.

Daß die Quinte zumindest als Kandidat in die engere Wahl fällt, darüber wird kaum Dissens bestehen. Der interessante und sensible Punkt ist daher die Wahl eines zweiten Basistonvektors. Zwischen den Terzen, denen die Funktionstheorie eine eigene Dimension zuerkennt, und den akzidentiellen Tönen, denen die Stufenlehre eine eigene Dimension eher verwehrt als zugesteht, besteht ein enger Zusammenhang. So geht schon bei der Herausbildung der Vokalpolyphonie seit dem 13. Jahrhundert die Aufwertung der Terzen und Sexten in den Rang der Konsonanzen einher mit der zunehmenden Verwendung von Akzidenzien in Ergänzung des Vorrats an diatonischen Stammtönen. Typischerweise sind es Terzen oder Sexten, welche, einen akzidentiellen Ton enthaltend, den perfekten Schlußkonsonanzen vorangehen. Bereits die beiden das ^Hexachord naturale s erweiternden Töne $b$ und $h$ haben je nach Kontext Stammton- oder Akzidenzcharakter. Um der besonderen Rolle akzidentieller Nicht-Stammtöne gerecht zu werden, ist es durchaus naheliegend, ihnen Positionen außerhalb der diatonischen Stammtonachse zuzuweisen.

B

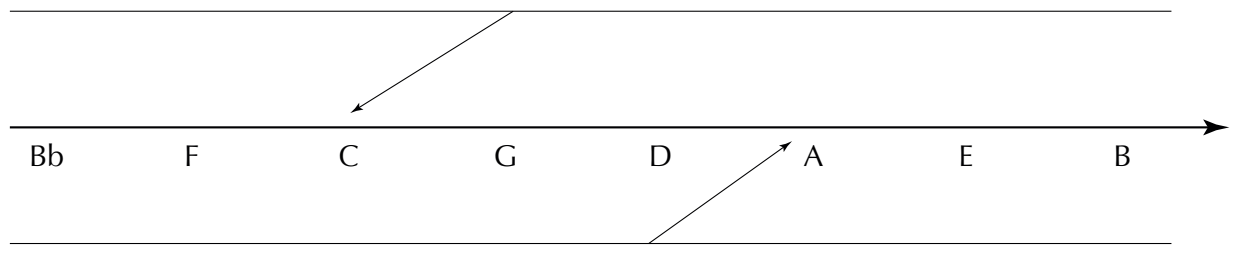


Während Funktionstheoretiker zweifellos zugunsten der Terz als zweitem Basistonvektor neben der Quinte argumentiert haben würden, hätten Anhänger der Stufenlehre wohl am ehesten die ‘Leitseptime r gewählt, also jenen Tonvektor, dessen Diskant der untere Leitton zum Grundton ist. Beide Ansätze führen jedoch nicht zu den aufschlußreichen Ergebnissen, die man erhält, wenn man einen anderen Basistonvektor neben die Quinte stellt: die `Leitquarte`.

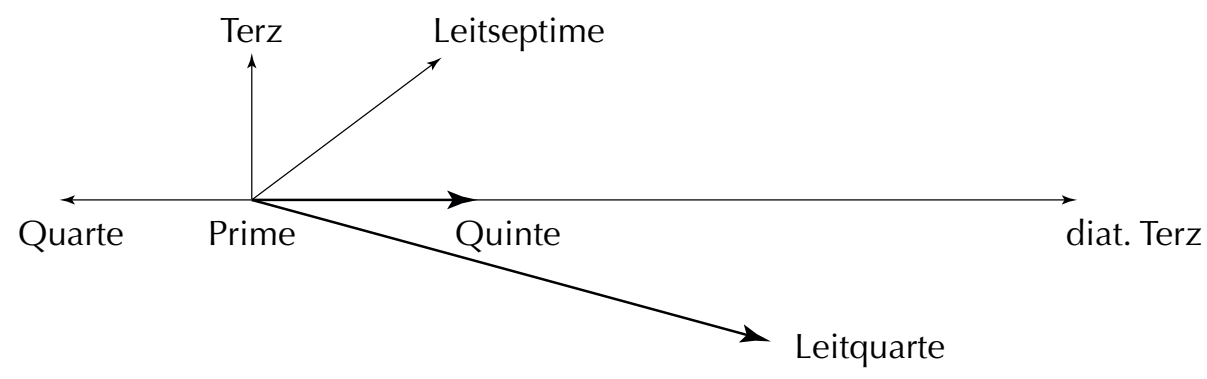

- In Hinlick auf die Alte Musik erscheint die Leitquarte (genauer: ihr Diskant) als oberer Leitton zur diatonischen Terz (genauer: zu ihrem Diskant). Die Leitquarte würde sich für die alte Musik dann als wichtig erweisen, wenn man eine höhere Beweglichkeit im Verhältnis von Stammtönen und akzidentiellen Tönen in Betracht zieht. Eine Konsequenz der Homogenität des Tonvektorgitters ist ja, daß sich auf den akzidentiellen Gitterlinien auch akzidentielle Varianten der Stammtöne (genauer: derjenigen Tonvektoren, die die betreffenden Töne zum Diskant haben) finden lassen. Beispielsweise gilt es zu untersuchen, unter welchen Umständen etwa die Leittonfortschreitungen $m i \rightarrow f a$ bzw. fa $\rightarrow$ mi die Auffassung nahelegen, mi als Diskant der Terz bzw. fa als Diskant der Leitquarte zu deuten.

- Hinsichtlich der jüngeren tonalen Harmonik erlangt die Leitquarte interessanterweise gerade dann musiktheoretisch besondere Bedeutung, wenn man versucht, elementare Bausteine von Stufenlehre und Funktionstheorie miteinander zu verbinden: den Dreiklang der zweiten Stufe /I mit dem Dreiklang der Subdominantparallele $S p$. Nimmt man die Prominenz der Akkordfortschreitung $I I \rightarrow V$ ernst, wie ihn die Stufentheorie postuliert, und folgt man den Funktionstheoretikern mindestens in dem Punkt, das sich der Grundton des Dreiklanges der Subdominantparallele mit der Subdominantsexte (genauer: ihrem Diskant) deckt, dann ergibt sich aus der Kombination beider Forderungen eine Prominenz der Leitquarte über dem Grundton der Subdominantparallele, denn ihr Diskant ist der Grundton des Dominantdreiklanges.

Die nachstehende Abbildung zeigt eine modifizierte Anordnung des Tonvektorgitters, bei der die Quintachse nach rechts ausgerichtet ist und die der Leitquarte nach unten. Zur Vermeidung einer umständlichen Nomenklatur der Tonvektoren wurden die Gitterpunkte mit Tonnamen etikettiert. Dennoch sind die Punkte dieses Gitters als Tonvektoren anzusehen. 


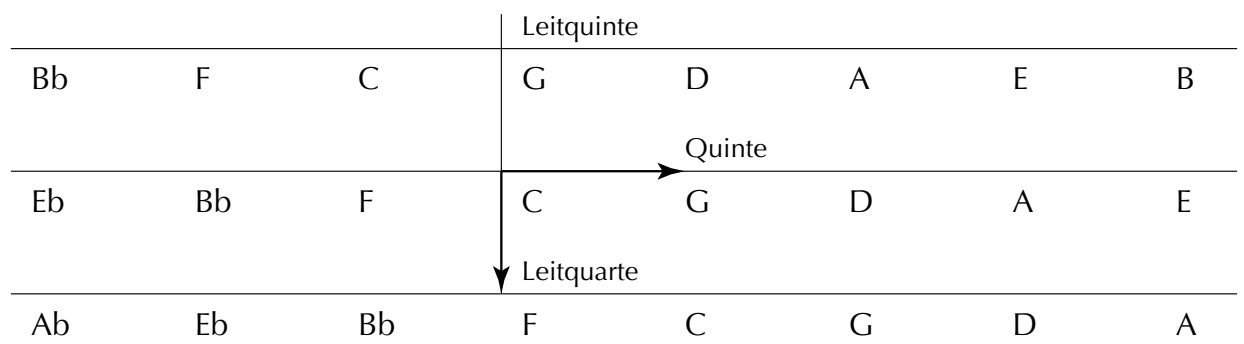

\subsection{Eulersche Apperzeptionspläne}

Zur Auszeichnung jener nur aus Quinte, Leitquarte, Quarte und Leitquinte zusammengesetzen Apperzeptionspläne sprechen wir von `Eulerschen Apperzeptionsplänen`, denn diese sind bis auf die Ersetzung der Terz durch die Leitquarte als Basistonvektor direkt aus dem Eulerschen Tonnetz abgeleitet. Die folgenden Berechnungen sind so dargestellt, daß sie auch ohne ein erschöpfendes Verständnis der im Abschnitt 2 erläuterten mathematischen Theorie nachvollziehbar sind.

Dazu geben wir die Berechnungsvorschriften der vier Apperzeptionen $T^{\triangleright}$ (Quinte), $T^{\triangleleft}$ (Quarte), $T^{\nabla}$ (Leitquarte) und $T^{\triangleleft}$ (Leitquinte) von einem beliebigen Tonort $T$ aus explizit an. Wir erinnern, daß Tonorte in der Standardbasis als $2 \times 2-$ Matrizen der Determinante 1 gegeben sind:

$$
T=\left(\begin{array}{ll}
a & b \\
c & d
\end{array}\right), \quad a d-b c=1 .
$$

Wir betrachten zuerst die Apperzeption des Tonvektors $q=\left(\begin{array}{ll}0 & 1 \\ 0 & 0\end{array}\right)$ an einem beliebigen Tonort $T$. Der Tonvektor $\mathrm{q}$ selbst ist auf den Zentralton bezogen und muß zunächst in den Tonort $T$ verschoben werden. Das Resultat dieser Verschiebung ist $q_{T}=\left(\begin{array}{ll}0 & a \\ 0 & c\end{array}\right)$. Da $q$ quintartig ist, ergibt sich die Apperzeption $T^{\triangleright}$ (nach Lemma 3) aus der Addition von $T$ und $q_{T}$ (in $\left.M_{2}(\mathbb{R})\right)$, d.h.

$$
T^{\triangleright}=\left(\begin{array}{ll}
a & b \\
c & d
\end{array}\right)^{\triangleright}=\left(\begin{array}{ll}
a & b+a \\
c & d+c
\end{array}\right)
$$

Das Resultat ist offensichtlich dasselbe wie nach der Apperzeptionsformel:

$$
\operatorname{App}(T, q)=T \cdot \operatorname{Exp}(q)=\left(\begin{array}{ll}
a & b \\
c & d
\end{array}\right) \cdot\left(\begin{array}{ll}
1 & 1 \\
0 & 1
\end{array}\right)=\left(\begin{array}{ll}
a & b+a \\
c & d+c
\end{array}\right) .
$$

Ganz analog erhält man in den anderen drei Fällen: 


$$
\begin{aligned}
& \left(\begin{array}{ll}
a & b \\
c & d
\end{array}\right)^{\triangleleft}=\left(\begin{array}{ll}
a & b-a \\
c & d-c
\end{array}\right), \\
& \left(\begin{array}{ll}
a & b \\
c & d
\end{array}\right)^{\nabla}=\left(\begin{array}{ll}
a+b & b \\
c+d & d
\end{array}\right), \\
& \left(\begin{array}{ll}
a & b \\
c & d
\end{array}\right)^{\nabla}=\left(\begin{array}{ll}
a-b & b \\
c-d & d
\end{array}\right) .
\end{aligned}
$$

\subsection{Tonverwandtschaften zweiten Grades}

Eine der Erwartungen an das Apperzeptionsmodell besteht in seiner Sensitivität gegenüber der Reihenfolge der Apperzeptionsschritte. Das Modell sollte so beschaffen sein, daß sich scheinbar zielgleiche Apperzeptionspläne, die traditionelle Tonverwandtschaften wie »Subdominante der Parallele« versus »Parallele der Sudominante« reflektieren, nicht nur in der Abfolge, sondern auch im Zieltonort unterscheiden sollten. Die Länge eines Apperzeptionsplans kann man mit dem durch die zugehörige Apperzeptionsbahn gestifteten Verwandtschaftsgrad assoziieren. Tonverwandtschaften zweiten Grades werden also durch Apperzeptionspläne der Länge 2 gestiftet.

Wir studieren nun die beiden Apperzeptionspläne $(-q, p)$ und $(p,-q)$. Sie sind scheinbar zielgleich mit dem gemeinsamen Zieltonvektor $p-q$. Wir beginnen mit der Beschreibung einer zu modellierenden Situation unter der getroffenen Einschränkung, daß Tonverwandtschaften nur mit Hilfe von Quinte, Leitquarte und der Umkehrintervalle Quarte und Leitquinte erfaßt werden können. Insbesondere stellt deshalb die Terzverwandtschaft keine Verwandtschaft ersten Grades dar. Im Lichte einer Unterscheidung von "Subdominante der Parallele« versus »Parallele der Sudominante« ist nicht der Schritt vom Grundton der Durtonika zur parallelen Molltonika elementar im Sinne einer Verwandtschaft ersten Grades, sondern vielmehr der Schritt von der Tonikaquinte zum Grundton der Subdominate der Mollparallele.

Wir fixieren den Ton $D$ als Ausgangston und deuten nun den scheinbar identischen Ganztonschritt $D \rightarrow C$ auf zwei verschiedene Weisen als Tonverwandtschaft deuten. Den Zielton $C$ wählen wir in Bezug auf den Ausgangston $D$ nach folgenden Bedingungen:

1. Faßt man $C$ als Grundton der Tonika $C-E-G$ in $C$-Dur auf, dann soll unser Ausgangston $D$ der Grundton der Parallele $D-F-A$ der Subdominate $F-A-C$ sein.

2. Faßt man $C$ als Quinte der Tonika $F-A-C$ in $>$ F-Dur auf, dann soll unser Ausgangston $D$ die Quinte der Subdominate $G-B_{b}-D$ der Parallele $D-F-A$ der Tonika $F-A-C$ sein. 
Während beide Bestimmungen im Eulergitter zum selben Ton führen, stimmen die Zieltonorte der Apperzeptionspläne $(-q, p)$ und $(p,-q)$ nicht überein:

$$
\begin{aligned}
T^{\nabla \triangleleft} & =\left(\begin{array}{ll}
a & b \\
c & d
\end{array}\right)^{\nabla \triangleleft}=\left(\begin{array}{ll}
a+b & b \\
c+d & d
\end{array}\right)^{\triangleleft}=\left(\begin{array}{ll}
a+b & -a \\
c+d & -c
\end{array}\right), \\
T^{\triangleleft \nabla} & =\left(\begin{array}{ll}
a & b \\
c & d
\end{array}\right)^{\triangleleft \nabla}=\left(\begin{array}{ll}
a & b-a \\
c & d-c
\end{array}\right)^{\nabla}=\left(\begin{array}{ll}
b & b-a \\
d & d-c
\end{array}\right) .
\end{aligned}
$$

Die Apperzeptionspläne $(-q, p)=$ (Leitquarte, Quarte) und $(p,-q)=($ Quarte, Leitquarte) sind also sscheinbar zielgleich, aber nicht zielgleichı. Für den zentralen Tonort $Z$ ergibt sich:

$$
Z^{\nabla \triangleleft}=\left(\begin{array}{cc}
1 & -1 \\
1 & 0
\end{array}\right) \quad \text { und } \quad Z^{\triangleleft \nabla}=\left(\begin{array}{cc}
0 & -1 \\
1 & 1
\end{array}\right) \text {. }
$$

Wählt man den Tonort $Z$ als Ausgangston $D$ unseres Beispiels, so ergeben sich für die beiden Varianten des Zieltones $C$ die Tonorte $Z^{\nabla}$ und $Z^{\nabla}$. Wählt man umgekehrt den zentralen Tonort $Z$ als festen Zielton $C$, so hat man es nur scheinbar mit einem Ausgangston $D$ zu tun, dem die beiden folgenden Tonorte entsprechen:

$$
D_{1}=Z^{\triangleright \Delta}=\left(\begin{array}{cc}
0 & 1 \\
-1 & 1
\end{array}\right) \text { und } D_{2}=Z^{\Delta \triangleright}=\left(\begin{array}{cc}
1 & 1 \\
-1 & 0
\end{array}\right)
$$

Für diese gilt dann:

$$
\begin{aligned}
& D_{1}^{\nabla \triangleleft}=\left(Z^{\triangleright \Delta}\right)^{\nabla \triangleleft}=Z^{\triangleright(\Delta \nabla) \triangleleft}=Z^{\triangleright \triangleleft}=Z, \\
& D_{2}^{\triangleleft \nabla}=\left(Z^{\Delta \triangleright}\right)^{\triangleleft \nabla}=Z^{\Delta(\triangleright \triangleleft) \nabla}=Z^{\Delta \nabla}=Z .
\end{aligned}
$$

\subsection{Die syntonische Identifikation}

Wenn wir das syntonische Komma von seiner Bedeutung als Tonhöhendifferenz befreien, so bleibt die Auffassung als Intervall, oder genauer: als Tonvektor $q+p$ (Quinte + Leitquarte). Angeregt durch Hugo Riemanns Argumentation hatten wir im Abschnitt 1 die abstrakte Forderung aufgestellt, daß dieses Intervall nicht apperzipierbar sein soll. Im Rahmen des mathematischen Modells werden Tonvektoren in Abhängigkeit vom Vorzeichen ihrer dia-syntonischen Norm in diatonartige, quintartige und syntonartige unterschieden. Der syntonische Tonvektor $\sigma_{1}=q+p$ ist in diesem Sinne syntonartig, denn es gilt $\left\|\sigma_{1}\right\|=-1$. Das ıApperzeptionspostulat, das prinzipiell das Apperzipieren synton-artiger Tonvektoren ausschließt, enthält unsere Interpretation der Riemannschen Aussagen als Spezialfall. 
Dieses generelle Postulat kann allerdings erst durch eine eingehendere musiktheoretische Diskussion der dia-syntonischen Norm gerechtfertigt werden. ${ }^{8}$

Zu den besonderen Eigenschaften des mathematischen Modells gehört die Nichttransitivität der Apperzeption. Erstens ist nicht jede Apperzeptionsbahn schritthaft, d.h. ihr Zieltonort ist im allgemeinen nicht bereits durch eine einzelne Apperzeption erreichbar. Zweitens gibt es schritthafte Apperzeptionsbahnen, die das Apperzeptionspostulat erfüllen und dennoch syntonartig sind. Die zugehörigen Apperzeptionspläne unterscheiden sich dann unmerklich von einer unmittelbaren Verletzung des Apperzeptionspostulats. Aus der Sicht des Ausgangstonortes hat man dabei den Eindruck, daß der Apperzeptionskegel mit einem Schritt verlassen wird.

Alle musiktheoretischen Phänomene, die auf dieser Tatsache beruhen, sollen unter dem Begriff der sSyntonizität zusammengefaßt werden. Als bislang einziges, aber musiktheoretisch zentrales Phänomen dieser Art behandeln wir nun die syntonische Identifikation.

Wir beginnen diese Untersuchung mit der Feststellung, daß unser Interesse nicht nur dem syntonischen Tonvektor $q+p$ selbst, oder genauer: dem aus diesem Vektor bestehenden Apperzeptionsplan $(q+p)$ gilt, sondern auch den beiden $z u(q+p)$ scheinbar zielgleichen Apperzeptionsplänen $(q, p)$ und $(p, q)$. Wenn man in Rechnung stellt, daß syntonische Identifikation sehr oft im Kontext einer Quint-Leitquart-Verwandtschaft oder Leitquart-Quint-Verwandtschaft auftritt, verdienen letztere aus musiktheoretischer Sicht sogar das größere Interesse. Die Zieltonorte aller drei Apperzeptionspläne (vom Zentralton $Z$ aus) sind sämtlich verschieden, d.h., es handelt sich um scheinbare, aber nicht um echte Zielgleichheit. Die erste dieser drei Apperzeptionsbahnen, der Apperzeptionsschritt $(Z, \operatorname{App}(Z, q+p))$ verstößt gegen das Apperzeptionspostulat.

$$
\begin{aligned}
\operatorname{App}(Z, q+p) & =\left(\begin{array}{cc}
\cosh (1) & \sinh (1) \\
\sinh (1) & \cosh (1)
\end{array}\right) \\
Z^{\triangleright \nabla} & =\left(\begin{array}{ll}
1 & 1 \\
0 & 1
\end{array}\right)^{\nabla}=\left(\begin{array}{ll}
2 & 1 \\
1 & 1
\end{array}\right) \\
Z^{\nabla \triangleright} & =\left(\begin{array}{ll}
1 & 0 \\
1 & 1
\end{array}\right)^{\triangleright}=\left(\begin{array}{ll}
1 & 1 \\
1 & 2
\end{array}\right)
\end{aligned}
$$

Die beiden Apperzeptionsbahnen $\left(Z, Z^{\triangleright}, Z^{\triangleright \nabla}\right)$ und $\left(Z, Z^{\nabla}, Z^{\nabla \triangleright}\right)$ sind nur unmerklich verschieden von einem (unmittelbaren) Verstoß gegen das Apperzeptionspostulat, denn sie

8 Es besteht eine formale Analogie zwischen der Behandlung der Apperzeptibilität in unserem Modell einerseits und der Behandlung von Kausalität im Rahmen der speziellen Relativitätstheorie andererseits. Wertvolle Anregungen hierzu verdanken wir der Kognitionsauffassung Michael Leytons [6]. Diese Analogie sollte nicht zur Rechtfertigung musiktheoretischer Postulate herangezogen werden. Die Brauchbarkeit analoger Übertragung muß sich auch daran messen lassen, ob mit ihrer Hilfe innerhalb der Musiktheorie weitere Phänomene gefunden werden können, die ihrerseits jene Rechtfertigung zu erbringen imstande sind. 
sind schritthaft und als solche syntonartig, d.h., für (p, q) und ( $q, p)$ gibt es zielgleiche Apperzeptionsschritte, die gegen das Apperzeptionspostulat verstoßen.

Diese Feststellungen sind bislang bloße Charakterisierungen der Apperzeptionspläne $(q+p),(q, p)$ und $(p, q)$. Über das Phänomen der syntonischen Identifikation im Sinne einer Vernachlässigung des syntonischen Intervalls ist damit noch nichts gesagt.

Wir greifen nun die beiden Apperzeptionspläne $(-p, q)$ und $(q,-p)$ (siehe 3.1) auf und erweitern sie je um einen weiteren Schritt zu: $P_{1}=(-q, p,-q)$ und $P_{2}=(p,-q, p)$. Diese beiden Apperzeptionspläne $P_{1}$ und $P_{2}$ sind offensichtlich nicht scheinbar zielgleich, da sich ihre Zieltonvektoren $-2 q+p$ und $2 p-q$ um den syntonischen Tonvektor unterscheiden: $(2 p-q)-(-2 q-p)=q+p$. Wie die folgende Rechnung zeigt, sind die Apperzeptionspläne $P_{1}$ und $P_{2}$ jedoch zielgleich.

$$
\begin{aligned}
& T^{\triangleleft \nabla \triangleleft}=\left(\begin{array}{ll}
a & b \\
c & d
\end{array}\right)^{\triangleleft \nabla \triangleleft}=\left(\begin{array}{ll}
a & b-a \\
c & d-c
\end{array}\right)^{\nabla \triangleleft}=\left(\begin{array}{ll}
b & b-a \\
d & d-c
\end{array}\right)^{\triangleleft}=\left(\begin{array}{ll}
b & -a \\
d & -c
\end{array}\right) \\
& T^{\nabla \triangleleft \nabla}=\left(\begin{array}{ll}
a & b \\
c & d
\end{array}\right)^{\nabla \triangleleft \nabla}=\left(\begin{array}{ll}
a+b & b \\
c+d & d
\end{array}\right)^{\triangleleft \nabla}=\left(\begin{array}{ll}
a+b & -a \\
c+d & -c
\end{array}\right)^{\nabla}=\left(\begin{array}{ll}
b & -a \\
d & -c
\end{array}\right)
\end{aligned}
$$

Damit haben wir zwei unmerklich verschiedene Apperzeptionspläne gefunden, deren Unterschied gerade der syntonische Tonvektor ist. Die folgende Gleichung nennen wir deshalb die ssyntonische Identifikation<.

$$
\triangleleft \nabla \triangleleft=\nabla \triangleleft \nabla
$$

Wir betrachten nun noch äquivalente Umformungen dieser Gleichung.

$$
\begin{gathered}
\triangleright(\triangleleft \nabla \triangleleft) \triangle=\triangleright(\nabla \triangleleft \nabla) \triangle \\
(\triangleright \triangleleft) \nabla \triangleleft \triangle=\triangleright \nabla \triangleleft(\nabla \triangle) \\
\nabla \triangleleft \triangle=\triangleright \nabla \triangleleft
\end{gathered}
$$

Die letzte Gleichung besagt in mathematischer Terminologie, daß die Quartapperzeption $\triangleleft$ und die Leitquartapperzeption $\nabla$ je Konjugierte $\nabla \triangleleft \Delta$ und $\triangleright \nabla \triangleleft$ besitzen, die untereinander gleich sind. Solche Konjugationen lassen sich auf einer Metaebene als »Perspektivenwechsel« auffassen. Quartapperzeption und Leitquartapperzeption stimmen danach bis auf einen inneren Wechsel der Perspektiven überein. Eine weitere äquivalente Umformung ergibt:

$$
\begin{gathered}
\nabla \triangleleft \Delta \triangleright=\triangleright \nabla(\triangleleft \triangleright), \\
\nabla \triangleleft \triangle \triangleright=\triangleright \nabla .
\end{gathered}
$$

Die rechte Seite der zweiten Gleichung entspricht nun genau dem Apperzeptionsplan $(q, p)$. Ihre linke Seite entspricht dem scheinbar trivialen Apperzeptionsplan 
$(p,-q,-p, q)$ mit dem Zieltonvektor 0. Damit läßt sich das Phänomen der syntonischen Identifikation im Kontext unseres Theorieentwurfes wie folgt charakterisieren:

Der Apperzeptionsplan ( $q, p)$, der vorsieht, daß nacheinander Quinte und Leitquarte apperzipiert werden, ist

1. unmerklich verschieden von einer Verletzung des Apperzeptionspostulats durch einen direkten syntonartigen Apperzeptionsschritt und

2. unmerklich verschieden von einer scheinbar trivialen Apperzeption.

\subsection{Die enharmonischen Identifikationen}

Unser Interesse gilt nunmehr den Apperzeptionsplänen, die unmerklich verschieden von der (echten) trivialen Apperzeption sind. Wir nennen sie senharmonische Identifikationen . Die Identifikation, die dabei vorgenommen wird, ist die zwischen dem Zieltonvektor des Apperzeptionsplans und dem Primvektor. Eine systematische Bestimmung aller enharmonischen Identifikationen ist eine gruppentheoretisch anspruchsvolle Aufgabe. Wir begnügen uns hier mit der Vorstellung dreier musiktheoretisch prominenter Fälle.

Wir betrachten zuerst den Apperzeptionsplan

$$
P=(p,-q, p,-q, p,-q, p,-q, p,-q, p,-q) \text {. }
$$

Es handelt sich um einen wiederholten Wechsel von Quart- und Leitquartapperzeption, wie er etwa buchstäblich in den Grundtönen folgender $I I-V$ - Sequenz auftritt. $F_{\#} m^{7}$ $\begin{array}{llllllllllll}B^{7} & \mathrm{Em}^{7} & A^{7} & \mathrm{Dm}^{7} & G^{7} & \mathrm{Cm}^{7} & F^{7} & B_{b} m^{7} & E_{b}^{7} & A_{b} m^{7} & D_{b}^{7} & \left(G_{b}\right) \text {. Sein scheinbarer Ziel- }\end{array}$ tonvektor ist $6 p-6 q$. Wie die folgende Rechnung zeigt, ist dieser Apperzeptionsplan jedoch zielgleich mit der trivialen Apperzeption:

$$
\begin{aligned}
& \left(\begin{array}{ll}
a & b \\
c & d
\end{array}\right)^{\nabla \triangleleft \nabla \triangleleft \nabla \triangleleft \nabla \triangleleft \nabla \triangleleft \nabla \triangleleft}=\left(\begin{array}{ll}
a+b & b \\
c+d & d
\end{array}\right)^{\triangleleft \nabla \triangleleft \nabla \triangleleft \nabla \triangleleft \nabla \triangleleft \nabla \triangleleft} \\
& =\left(\begin{array}{ll}
a+b & -a \\
c+d & -c
\end{array}\right)^{\nabla \triangleleft \nabla \triangleleft \nabla \triangleleft \nabla \triangleleft \nabla \triangleleft}=\left(\begin{array}{cc}
b & -a \\
d & -c
\end{array}\right)^{\triangleleft \nabla \triangleleft \nabla \triangleleft \nabla \triangleleft \nabla \triangleleft} \\
& =\left(\begin{array}{ll}
b & -a-b \\
d & -c-d
\end{array}\right)^{\nabla \triangleleft \nabla \triangleleft \nabla \triangleleft \nabla \triangleleft}=\left(\begin{array}{ll}
-a & -a-b \\
-c & -c-d
\end{array}\right)^{\triangleleft \nabla \triangleleft \nabla \triangleleft \nabla \triangleleft} \\
& =\left(\begin{array}{ll}
-a & -b \\
-c & -d
\end{array}\right)^{\nabla \triangleleft \nabla \triangleleft \nabla \triangleleft}=\left(\begin{array}{ll}
-a-b & -b \\
-c-d & -d
\end{array}\right)^{\triangleleft \nabla \triangleleft \nabla \triangleleft} \\
& =\left(\begin{array}{ll}
-a-b & a \\
-c-d & c
\end{array}\right)^{\nabla \triangleleft \nabla \triangleleft} \quad=\left(\begin{array}{ll}
-b & a \\
-d & c
\end{array}\right)^{\triangleleft \nabla \triangleleft}
\end{aligned}
$$




$$
\begin{aligned}
& =\left(\begin{array}{ll}
-b & a+b \\
-d & c+d
\end{array}\right)^{\nabla \triangleleft} \quad=\left(\begin{array}{ll}
a & a+b \\
c & c+d
\end{array}\right)^{\triangleleft} \\
& =\left(\begin{array}{ll}
a & b \\
c & d
\end{array}\right) .
\end{aligned}
$$

Als zweiten Fall, der jene von Pfrogner und Berlioz thematisierte Situation betrifft, betrachten wir die enharmonische Identifikation von $G_{b}$ (als Terz der Subdominante in $B_{b}$

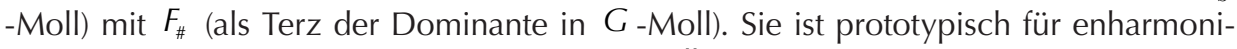
sche Modulationen von $B_{b}-$ Moll nach $G-M o l l$, und sie tritt auch an jener berühmten von Berlioz/Strauss explizit besprochenen Stelle in Glucks »Orpheus« auf, wo beide Töne sogar gleichzeitig präsent sind. Als Apperzeptionsplan kommt dabei eine dreifache Iteration des Terzapperzeptionsplans $(q, q, q,-p)$ in Betracht:

$$
Q=(q, q, q,-p, q, q, q,-p, q, q, q,-p) .
$$

Der Zieltonvektor ist 9q-3p. Auch hier zeigt sich die Zielgleichheit des Apperzeptionsplans Q mit der trivialen Apperzeption:

$$
\begin{aligned}
& \left(\begin{array}{ll}
a & b \\
c & d
\end{array}\right)^{\triangleright \triangleright \triangleright \Delta \triangleright \triangleright \triangleright \Delta \triangleright \triangleright \triangleright}=\left(\begin{array}{ll}
a & b+3 a \\
c & d+3 c
\end{array}\right)^{\Delta \triangleright \triangleright \triangleright \Delta \triangleright \triangleright \triangleright \Delta} \\
= & \left(\begin{array}{ll}
-2 a-b & b+3 a \\
-2 c-d & d+3 c
\end{array}\right)^{\triangleright \triangleright \triangleright \Delta \triangleright \triangleright \triangleright}=\left(\begin{array}{ll}
-2 a-b & -2 b-3 a \\
-2 c-d & -2 d-3 c
\end{array}\right)^{\Delta \triangleright \triangleright \triangleright \Delta} \\
= & \left(\begin{array}{ll}
a+b & -2 b-3 a \\
c+d & -2 d-3 c
\end{array}\right)^{\triangleright \triangleright \triangleright \Delta}=\left(\begin{array}{ll}
a+b & b \\
c+d & d
\end{array}\right)^{\Delta} \\
= & \left(\begin{array}{ll}
a & b \\
c & d
\end{array}\right) .
\end{aligned}
$$

Anhand des Apperzeptionsplans

$$
(q, q, q, q, q, q, q, q, q, q, q, q)
$$

mit dem Zieltonvektor 12q und dem Apperzeptionsziel

$$
\left(\begin{array}{ll}
a & b \\
c & d
\end{array}\right)^{\Delta \triangleright \triangleright \triangleright \triangleright \triangleright \triangleright \triangleright \triangleright \triangleright \triangleright \triangleright \triangleright}=\left(\begin{array}{ll}
a & b+12 a \\
c & d+12 c
\end{array}\right)
$$

wird schließlich deutlich, daß nicht jeder enharmonischen Verwechslung im traditionellen Sinne auch eine enharmonische Identifikation im Rahmen unseres Modells entspricht. Die Tonvektorinterpretation $12 q$ des spythagoräischen Komması wäre dazu ein 
Gegenbeispiel. Die beiden nun folgenden Paragraphen gehen den sich daraus ergebenden Fragen in zweierlei Richtung nach.

\subsection{Enharmonische Verwechslung}

Im Zusammenhang mit der enharmonischen Identifikation verweisen wir auf zwei wichtige mathematische Tatsachen, die mit der Gruppenstruktur von $S L_{2}(\mathbb{Z})$ aller $2 \times 2$-Matrizen $\left(\begin{array}{ll}a & b \\ c & d\end{array}\right)$ mit ganzzahligen Koeffizienten $a, b, c, d, a d-b c=1$, zusammenhängen. Die zugehörigen Tonorte nennen wir `Eulersche Tonorte und verwenden den traditionellen Begriff des `Tonnetzes für den von ihnen gebildeten Raumı.

- Für jeden Eulerschen Tonort $T$ gibt es einen Eulerschen Apperzeptionsplan (der also nur aus Leitquart-, Quart-, und Quintschritten besteht) derart, daß T das Apperzeptionsziel dieses Apperzeptionsplans vom zentralen Tonort $Z$ aus ist.

- Identifiziert man je die Zieltöne aller scheinbar zielgleichen Eulerschen Apperzeptionspläne vom zentralen Tonort aus (im strengen Sinne einer Äquivalenzrelation), so ergeben sich aus der Gesamtheit aller Eulerschen Apperzeptionspläne genau zwölf Äquivalenzklassen. ${ }^{9}$

Jene »Identifikation« von Zieltönen zu Äquivalenzklassen ist eine Operation auf der Metaebene der Untersuchung und keine Identifikation durch Apperzeption innerhalb des Modells wie im Falle der bereits besprochenen syntonischen und enharmonischen Identifikation. Die Transitivität dieser Äquivalenzrelation führt auf eine erstaunlich einfache Modellierung des Begriffs der senharmonischen Verwechslung`: Sie beruht auf der beliebigen Verwechslung von scheinbarer Zielgleichheit und echter Zielgleichheit Eulerscher Apperzeptionspläne, und zwar in beiden Richtungen. Dies ist ein Phänomen auf der Metaebene, da eine solche Verwechslung zwischen scheinbarer und echter Zielgleichheit im allgemeinen nur als eine ^Verwechslung von Apperzeptionsbahnen beschrieben werden kann, nicht aber als eine Apperzeptionsbahn selbst. ${ }^{10}$

Eine generelle Möglichkeit zur enharmonischen Verwechslung von Apperzeptionsbahnen ersieht man aus einer weiteren äquivalenten Umformung der Gleichung $\nabla \triangleright \triangle \triangleright=\triangleright \nabla$

$$
\triangleleft(\nabla \triangleleft \triangle \triangleright)=\nabla
$$

9 Dahinter steht die gruppentheoretische Tatsache, daß die Faktorgruppe von $S L_{2}(\mathbb{Z})$ nach ihrer Kommutatoruntergruppe eine zyklische Gruppe der Ordnung 12 ist (Noll 1997, 123).

10 Eine Theorie, die darauf abzielt, ausgehend vom vorgestellten Apperzeptionsmodell enharmonische Verwechslungen als tatsächliche Denkvorgänge zu beschreiben, sieht sich mit dem Problem konfrontiert, daß die Apperzeption nicht nur ihre infinitesimalen` Pläne zu redigieren hätte, sondern ihre Bahnen selbst, und damit die Substanz ihrer eigenen Prozeßgeschichte. Damit wäre Apperzeption vielleicht als Überlagerung mehrerer oder aller Möglichkeiten aufzufassen. 
Die letzte Gleichung drückt die Zielgleichheit der Apperzeptionspläne $(-q, p, q,-p, q)$ und $(p)$ aus. Andererseits ist $\Sigma(-q, p,-q,-p, q)=-q$, woraus sich die scheinbare Zielgleichheit der Apperzeptionspläne $(-q, p,-q,-p, q)$ und $(-q)$ ergibt. Erlaubt man nun die Verwechslung der scheinbaren mit der echten Zielgleichheit, so ergibt sich damit die enharmonische Verwechselbarkeit der Apperzeption von Quarte $-q$ und Leitquarte $p$. Damit erweist sich die enharmonische Verwechslung im Sinne unserer Definition eigentlich als eine »syntonische Verwechslung«: In jedem Apperzeptionsplan können -q gegen $p$ bzw. $q$ gegen $-p$ ausgetauscht werden. Wie man aber sofort sieht, umfaßt unser Begriff der enharmonischen Verwechslung auch jede im üblichen Sinne: Durch die syntonische Verwechslung von drei Tonvektoren im Apperzeptionsplan

$$
(q, q, q, q, q, q, q, q, q, q, q, q)
$$

entsteht der Apperzeptionsplan

$$
(q, q, q,-p, q, q, q,-p, q, q, q,-p)
$$

Dieser ist aber unmerklich verschieden von der Prime.

Dieser enge Zusammenhang von syntonischer und enharmonischer Identifikation bzw. Verwechslung wirft ein neues Licht auf die stabile Symbiose von Notenschrift und Zwölfteilung der Oktave auf den Tasteninstrumenten. Es scheint, daß Hermann Pfrogners einheitliche Sicht auf Diatonik, Chromatik und Enharmonik eine neue argumentative Grundlage erhält.

\subsection{Diatonartige Apperzeptionen}

Bei der Untersuchung der syntonischen Identifikation wurden syntonartige schritthafte Apperzeptionspläne diskutiert. Mit unserer Charakterisierung des Apperzeptionsplans $(q, p)$ als unmerklich verschieden von einem Verstoß gegen das Apperzeptionspostulat wurde den zugehörigen zielgleichen nicht apperzibierbaren Tonvektoren musiktheoretisch Bedeutung zugewiesen.

Um so zwingender ist Klärung der musiktheoretischen Bedeutung jener diatonartigen Tonvektoren, die zielgleich zu schritthaften Apperzeptionsplänen sind. Ohne einer genaueren Untersuchung vorzugreifen, sei auf die besondere Rolle der imaginären diatonischen Achse verwiesen, die sich im Zusammenhang mit der dia-syntonischen Norm ergibt.

Der Apperzeptionsplan $P=(p,-q, p,-q, p,-q, p,-q, p,-q, p,-q)$ enthält vier schritthafte Teilpläne, die jeweils zielgleich zur Apperzeption eines geeigneten imaginären diaton-artigen Tonvektors sind:

$$
\begin{aligned}
P_{E b} & =(p,-q, p), \\
P_{G b} & =(p,-q, p,-q, p,-q), \\
P_{B b b} & =(p,-q, p,-q, p,-q, p,-q, p), \\
P_{D b b} & =(p,-q, p,-q, p,-q, p,-q, p,-q, p,-q) .
\end{aligned}
$$


Explizit gilt:

$$
\begin{array}{ll}
T^{\nabla \triangleleft \nabla} & =\operatorname{App}\left(T,-\frac{\pi}{2} \delta_{1}\right), \\
T^{\nabla \triangleleft \nabla \triangleleft \nabla \triangleleft} & =\operatorname{App}\left(T,-\pi \delta_{1}\right), \\
T^{\nabla \triangleleft \nabla \triangleleft \nabla \triangleleft \nabla \triangleleft \nabla} & =\operatorname{App}\left(T,-\frac{3 \pi}{2} \delta_{1}\right) \\
T^{\nabla \triangleleft \nabla \triangleleft \nabla \triangleleft \nabla \triangleleft \nabla \triangleleft \nabla \triangleleft} & =A p p\left(T,-2 \pi \delta_{1}\right) .
\end{array}
$$

Es handelt sich um die Tonorte eines »verminderten Septakkordes«. Damit wird nahegelegt, auch den Tonvektoren $-\frac{\kappa \pi}{2} \delta_{1}, \kappa \in \mathbb{Z}$ musiktheoretische Bedeutung zuzuweisen. Via Zieltongleichkeit sind sie die Bindeglieder zwischen den bislang betrachteten Eulerschen Apperzeptionsplänen und einer unendlichen »temperierten« imaginären Quintenkette, $\frac{k \pi}{6} \delta_{1}, k \in \mathbb{Z}$, deren diatonartige Apperzeptionen ein zyklisches System von 12 Tonorten ergeben.

Schluß: Der Theorieentwurf mag Anlaß zu weiterführenden Spekulationen über das Tondenken und die Geistestätigkeit im allgemeinen geben. Allein, sie sind müßig, solange nicht innerhalb der Musiktheorie weitere Vorhersagen getroffen und geprüft worden sind. Die computergestützte korpusbasierte Analyse bietet hierzu ein breites Experimentierfeld an. Was die Spekulationen angeht, so halten wir uns an die epistemologische Bescheidenheit, die in Christian Morgensterns Lied vom blonden Korken ausgedrückt wird. ${ }^{11}$

Ein blonder Korke spiegelt sich

in einem Lacktablett -

allein er säh sich dennoch nich,

selbst wenn er Augen hätt!

Das macht, dieweil er senkrecht steigt

zu seinem Spiegelbild!

Wenn man ihn freilich seitwärts neigt,

zerfällt, was oben gilt.

O Mensch, gesetzt, du spiegelst dich

im, sagen wir, im All!

Und senkrecht! wärest du dann nich

ganz in demselben Fall?

Danksagung: Guerino Mazzola, Oliver Schwab-Felisch und Klaus Robering danken wir für anregende Disskussionen und vielfältige Hinweise, die dazu beitrugen, unseren Uberlegungen die jetzige Form zu geben.

11 Ein besonderer Reiz jenes Gleichnisses besteht in der impliziten kühnen Annahme, daß die Geometrie des Alls mit derjenigen des Menschen übereinstimmt. Immerhin: Die Quinte unseres Modells gleicht dem Korken insofern nicht, als sie auch ısenkrecht auf sich selbst steht. 


\section{Literatur}

Berlioz, Hector., Strauss, Richard: Instrumentationslehre, Edition Peters, Leipzig 1904.

Ebeling, Martin: Tonhöhe physikalisch - musikalisch - psychologisch - mathematisch, Peter Lang: Frankfurt/M 1998.

Gibbons, Gary W.: »Anti-de-Sitter Spacetime and Its Uses« In: Lecture Notes in Physics 537, Springer, Berlin 2000: 102-142.

Helgason, Sigurdur: Differential Geometry, Lie Groups, and Symmetric Spaces, Academic Press, New York 1978.

Iversen, Birger: Hyperbolic Geometry, London Mathematical Society Student Texts 25, Cambridge 1992.

Leyton, Michael: Symmetry, Causality, Mind, MIT Press, Massachusetts 1992.

Mazzola, Guerino: Die Geometrie der Töne, Birkhäuser, Basel 1990.

Mazzola, Guerino: The Topos of Music, Birkhäuser, Basel 2002.

Noll, Thomas: Morphologische Grundlagen der abendländischen Harmonik, Brockmeyer, Bochum 1997.

Pfrogner, Hermann: Die Zwölfordnung der Töne, Amalthea-Verlag, Zürich, 1953.

Riemann, Hugo: »Ideen zu einer Lehre von den Tonvorstellungen« in: Jahrbuch der Musikbibliothek Peters 21/22, Leipzig 1914/15, 1-26.

Vogel, Martin: Die Lehre von den Tonbeziehungen, Verlag für systematische Musikwissenschaft, Bonn 1975. 